\title{
Zero sets of eigenspinors for generic metrics
}

Andreas Hermann

Let $M$ be a closed connected spin manifold of dimension 2 or 3 with a fixed orientation and a fixed spin structure. We prove that for a generic Riemannian metric on $M$ the non-harmonic eigenspinors of the Dirac operator are nowhere zero. The proof is based on a transversality theorem and the unique continuation property of the Dirac operator.

$\begin{array}{llr}1 & \text { Introduction } & 178\end{array}$

$\begin{array}{llr}2 & \text { Preliminaries } & 179\end{array}$

$\begin{array}{lll}2.1 & \text { Review of spin geometry } & 179\end{array}$

$\begin{array}{lll}2.2 & \text { Further preliminaries } & 182\end{array}$

3 Green's function for the Dirac operator 183

3.1 The Bourguignon-Gauduchon trivialization 183

3.2 The Euclidean Dirac operator 186

$\begin{array}{lll}3.3 & \text { Expansion of Green's function } & 188\end{array}$

4 Zero sets of eigenspinors $\quad 196$

4.1 Eigenspinors in dimensions 2 and $3 \quad 196$

4.2 Examples on closed surfaces 215

$\begin{array}{lr}\text { Acknowledgments } & 216\end{array}$

$\begin{array}{lr}\text { References } & 216\end{array}$ 


\section{Introduction}

The spectrum of the Dirac operator can be computed explicitly for some closed Riemannian spin manifolds. In these examples, one very often has eigenvalues of high multiplicities (see e.g., $[9,14]$ ). However, it is also known that for a generic choice of metric on a closed spin manifold of dimension 2 or 3 the eigenvalues are simple (see [13]). In the present article we extend this result by showing that for a generic metric the non-harmonic eigenspinors are nowhere zero. More precisely let $M$ be a closed spin manifold and denote by $R(M)$ the space of all smooth Riemannian metrics on $M$ equipped with the $C^{1}$-topology. For every $g \in R(M)$ denote by $D^{g}$ the Dirac operator acting on spinors for the metric $g$. Let $N(M)$ be the subset of all $g \in R(M)$ such that all the eigenspinors of $D^{g}$ are nowhere zero on $M$ and let $N^{*}(M)$ be the subset of all $g \in R(M)$ such that all the non-harmonic eigenspinors of $D^{g}$ are nowhere zero on $M$. Then we prove the following.

Theorem 1.1. Let $M$ be a closed connected spin manifold with a fixed orientation and a fixed spin structure. If $\operatorname{dim} M=2$ then $N^{*}(M)$ is residual in $R(M)$. If $\operatorname{dim} M=3$ then $N(M)$ is residual in $R(M)$.

Recall that a subset is residual, if it contains a countable intersection of open and dense sets. The proof will show that for every $g \in R(M)$ the intersection of $N^{*}(M)$ with the conformal class $[g]$ of $g$ is residual in $[g]$ (see Theorem 4.3). In the case $\operatorname{dim} M=3$ we use the fact that the subset of all $g \in R(M)$ with $\operatorname{ker}\left(D^{g}\right)=\{0\}$ is open and dense in $R(M)$ (see [4, 23]). Furthermore, for $\operatorname{dim} M=2$ the subset $N(M)$ is in general not residual in $R(M)$. Namely in Section 4.2 we will give examples of closed surfaces $M$ such that for every metric $g$ on $M$ there exist harmonic spinors of $D^{g}$ with non-empty zero set. The proof will also show that Theorem 1.1 is true with respect to every $C^{k}$-topology, $k \geq 1$, on $R(M)$. In order to simplify the notation we will state all results using the $C^{1}$-topology.

In analysis, geometry and mathematical physics the study of zero sets of solutions to generalized Dirac equations is very important. On closed manifolds these zero sets have codimension 2 at least by a result of Bär (see [11]). There are many open questions in the literature concerning these zero sets. For example, Nester and his co-authors ask whether on a generic asymptotically Euclidean manifold of dimension 3 one can find a Witten spinor without zeros (see e.g., [16]). As another example we mention the question raised by Ammann, whether for a generic Riemannian metric on a closed spin manifold of dimension $n \geq 2$ one can find a nowhere vanishing 
spinor $\psi$ satisfying a non-linear equation of the form

$$
D^{g} \psi=C|\psi|^{2 /(n-1)} \psi, \quad C>0 \text { constant }
$$

$($ see $[2,3])$. We hope that some of the techniques developed in this article can be applied to these questions. However, we note that in both cases one has to consider non-conformal deformations of the metric, while in this article we consider mainly conformal deformations.

This article is organized as follows. In Section 2.1, we give a short review of spin geometry in order to fix the notation and we recall a method to compare spinors for different metrics. In Section 2.2, we state a transversality theorem from differential topology and Aronszajn's unique continuation theorem. These are the main tools for the proof of Theorem 1.1. We also need the expansion of Green's function for the operator $D^{g}-\lambda$ with $\lambda \in \mathbb{R}$ around the singularity. This expansion is derived in Section 3 . Then in Section 4.1 we prove Theorem 1.1. We first construct a continuous map $F$ which assigns to every Riemannian metric $h$ in an open neighborhood of a fixed metric $g$ an eigenspinor of $D^{h}$ viewed as a section of $\Sigma^{g} M$. Theorem 1.1 follows from the transversality theorem if we can prove that the evaluation map corresponding to $F$ is transverse to the zero section of $\Sigma^{g} M$. Assuming that this is not the case we obtain an equation involving Green's function for the operator $D^{g}-\lambda$ with $\lambda \in \mathbb{R}$. Using the expansion of this Green's function and using that $\lambda \neq 0$ we obtain a contradiction from the unique continuation theorem. Finally, in Section 4.2 we give an example showing that Theorem 1.1 does not hold for harmonic spinors on closed surfaces.

\section{Preliminaries}

\subsection{Review of spin geometry}

Let $(M, g)$ be an oriented Riemannian spin manifold of dimension $n$. We denote by $\mathrm{P}_{\mathrm{SO}}(M, g)$ the principal $\mathrm{SO}(n)$-bundle of positively oriented $g$-orthonormal frames. A spin structure on $(M, g)$ consists of a principal $\operatorname{Spin}(n)$-bundle $\mathrm{P}_{\text {Spin }}(M, g)$ and a two-fold covering

$$
\Theta: \quad \mathrm{P}_{\mathrm{Spin}}(M, g) \rightarrow \mathrm{P}_{\mathrm{SO}}(M, g),
$$

which is compatible with the group actions of $\mathrm{SO}(n)$ on $\mathrm{P}_{\mathrm{SO}}(M, g)$ and of $\operatorname{Spin}(n)$ on $\mathrm{P}_{\text {Spin }}(M, g)$. We will always assume that a spin manifold has 
a fixed orientation and a fixed spin structure. If in addition a Riemannian metric $g$ on $M$ is chosen, we denote the Riemannian spin manifold by $(M, g, \Theta)$. The spinor bundle is defined as the associated vector bundle $\Sigma^{g} M:=\mathrm{P}_{\text {Spin }}(M, g) \times_{\rho} \Sigma_{n}$, where $\rho$ is the spinor representation on the complex vector space $\Sigma_{n}$ of dimension $2^{[n / 2]}$. We will denote by $\langle.,$.$\rangle the usual$ Hermitian inner product on $\Sigma^{g} M$. The Levi-Civita connection on $(M, g)$ induces a connection on $\Sigma^{g} M$ which will be denoted by $\nabla^{g}$. For every $p \in M$ the Clifford multiplication by tangent vectors in $p$ will be denoted by

$$
T_{p} M \otimes \Sigma_{p}^{g} M \rightarrow \Sigma_{p}^{g} M, \quad X \otimes \psi \mapsto X \cdot \psi
$$

The Dirac operator $D^{g}$ is a linear elliptic differential operator of first order acting on smooth sections of $\Sigma^{g} M$ :

$$
D^{g}: \quad C^{\infty}\left(\Sigma^{g} M\right) \rightarrow C^{\infty}\left(\Sigma^{g} M\right)
$$

If $\left(e_{i}\right)_{i=1}^{n}$ is a local $g$-orthonormal frame of $T M$, then the Dirac operator is locally given by

$$
D^{g} \psi=\sum_{i=1}^{n} e_{i} \cdot \nabla_{e_{i}}^{g} \psi .
$$

Furthermore, for all $\psi \in C^{\infty}\left(\Sigma^{g} M\right)$ and for all $f \in C^{\infty}(M, \mathbb{C})$ we have

$$
D^{g}(f \psi)=\operatorname{grad}^{g}(f) \cdot \psi+f D^{g} \psi
$$

(see [21], p. 116). For a detailed introduction to the concepts of spin geometry which we use here see [21] or [15].

Let $(M, \Theta)$ be a closed spin manifold and let $g, h \in R(M)$. Then the spinor bundles $\Sigma^{g} M$ and $\Sigma^{h} M$ are two different vector bundles. The problem of identifying spinors and the Dirac operators $D^{g}$ and $D^{h}$ for the metrics $g$ and $h$ has been treated in the literature (see $[12,17,19,23]$ ) with the following result: there exists an isomorphism of vector bundles

$$
\beta_{g, h}: \quad \Sigma^{g} M \rightarrow \Sigma^{h} M
$$

which is a fiberwise isometry with respect to the inner products on $\Sigma^{g} M$ and $\Sigma^{h} M$. There also exists an isomorphism of vector bundles

$$
\bar{\beta}_{g, h}: \quad \Sigma^{g} M \rightarrow \Sigma^{h} M
$$

which induces an isometry of Hilbert spaces $L^{2}\left(\Sigma^{g} M\right) \rightarrow L^{2}\left(\Sigma^{h} M\right)$. It is obtained from $\beta_{g, h}$ by pointwise multiplication with a positive function which 
takes into account the change of the volume form. We have $\bar{\beta}_{h, g}=\bar{\beta}_{g, h}^{-1}$. By this isomorphism the Dirac operator $D^{h}$ can be regarded as a differential operator acting on spinors for the metric $g$. More precisely one defines

$$
D^{g, h}:=\bar{\beta}_{h, g} D^{h} \bar{\beta}_{g, h}: \quad C^{\infty}\left(\Sigma^{g} M\right) \rightarrow C^{\infty}\left(\Sigma^{g} M\right) .
$$

This operator has a self-adjoint closure in $L^{2}\left(\Sigma^{g} M\right)$.

For all $g, h \in R(M)$ there exists an open interval $I$ containing [0,1] such that for every $t \in I$ the tensor field $g_{t}:=g+t(h-g)$ is a Riemannian metric on $M$. This family of Riemannian metrics on $M$ induces real analytic families of eigenvalues and eigenspinors of the family of operators $\left(D^{g, g_{t}}\right)_{t \in I}$ (see [12]). Namely by a theorem of Rellich (see Theorem VII.3.9 in [20]) we have the following lemma.

Lemma 2.1. Let $\lambda$ be an eigenvalue of $D^{g}$ with $d:=\operatorname{dim}_{\mathbb{C}} \operatorname{ker}\left(D^{g}-\lambda\right)$. Then there exist real analytic functions

$$
I \ni t \mapsto \lambda_{j, t} \in \mathbb{R}, \quad 1 \leq j \leq d,
$$

such that for every $j$ and every the number $\lambda_{j, t}$ is an eigenvalue of $D^{g, g_{t}}$ and such that $\lambda_{j, 0}=\lambda$ for every $j$. Furthermore, there exist spinors $\psi_{j, t} \in$ $C^{\infty}\left(\Sigma^{g} M\right), 1 \leq j \leq d, t \in I$, which are real analytic in $t$, such that for every $t \in I$ the spinors $\psi_{j, t}, 1 \leq j \leq d$, form an $L^{2}$-orthonormal system and for every $t \in I$ and for every $j$ the spinor $\psi_{j, t}$ is an eigenspinor of $D^{g, g_{t}}$ corresponding to $\lambda_{j, t}$.

For all $j \in\{1, \ldots, d\}$ and for all $t \in I$ we have $\lambda_{j, t}=\left(\psi_{j, t}, D^{g, g_{t}} \psi_{j, t}\right)_{L^{2}}$. By taking the derivative at $t=0$ and using the fact that $D^{g}$ is self-adjoint and that for every $t$ we have $\left\|\psi_{j, t}\right\|_{L^{2}}=1$ we obtain

$$
\left.\frac{d \lambda_{j, t}}{d t}\right|_{t=0}=\int_{M}\left\langle\psi_{j, 0},\left.\frac{d}{d t} D^{g, g_{t}}\right|_{t=0} \psi_{j, 0}\right\rangle \mathrm{dv}^{g}, \quad 1 \leq j \leq d .
$$

If $g$ and $h$ are conformally related, i.e., $h=\mathrm{e}^{2 u} g$ for some function $u \in$ $C^{\infty}(M, \mathbb{R})$, then for all $\psi \in C^{\infty}\left(\Sigma^{g} M\right)$ we have

$$
D^{h}\left(\mathrm{e}^{-(n-1) u / 2} \beta_{g, h} \psi\right)=\mathrm{e}^{-(n+1) u / 2} \beta_{g, h} D^{g} \psi
$$

(see, e.g., [17]). Using that $\bar{\beta}_{g, h}=\mathrm{e}^{-n u / 2} \beta_{g, h}$ we have for all $\psi \in C^{\infty}\left(\Sigma^{g} M\right)$

$$
D^{g, h} \psi=\mathrm{e}^{-u / 2} D^{g}\left(\mathrm{e}^{-u / 2} \psi\right) .
$$


Let $f \in C^{\infty}(M, \mathbb{R})$ and let $I \subset \mathbb{R}$ be an open interval containing 0 such that for every $t \in I$ the tensor field $g_{t}:=g+t f g$ is a Riemannian metric on $M$. Then for all $\psi \in C^{\infty}\left(\Sigma^{g} M\right)$ we have

$$
D^{g, g_{t}} \psi=(1+t f)^{-1 / 4} D^{g}\left((1+t f)^{-1 / 4} \psi\right)
$$

and therefore

$$
\left.\frac{d}{d t} D^{g, g_{t}}\right|_{t=0} \psi=-\frac{1}{2} f D^{g} \psi-\frac{1}{4} \operatorname{grad}^{g}(f) \cdot \psi
$$

where the Clifford multiplication is taken with respect to the metric $g$. Using this formula in (2.1) and taking the real part we obtain

$$
\left.\frac{d \lambda_{j, t}}{d t}\right|_{t=0}=-\frac{\lambda}{2} \int_{M} f\left|\psi_{j, 0}\right|^{2} \mathrm{dv}^{g}, \quad 1 \leq j \leq d
$$

\subsection{Further preliminaries}

In this section, we briefly recall a transversality theorem from differential topology and a unique continuation theorem for generalized Laplace operators acting on sections of a vector bundle.

Definition 2.2. Let $f: Q \rightarrow N$ be a $C^{1}$ map between two manifolds. Let $A \subset N$ be a submanifold. $f$ is called transverse to $A$, if for all $x \in Q$ with $f(x) \in A$ we have

$$
T_{f(x)} A+\operatorname{im}\left(\left.d f\right|_{x}\right)=T_{f(x)} N
$$

We quote the following transversality theorem from [18, 24].

Theorem 2.3. Let $V, M, \Sigma$ be smooth manifolds and let $A \subset \Sigma$ be a smooth submanifold. Let $F: V \rightarrow C^{r}(M, \Sigma)$ be a map, such that the evaluation map $F^{\mathrm{ev}}: V \times M \rightarrow \Sigma,(v, m) \mapsto F(v)(m)$ is $C^{r}$ and transverse to A. If

$$
r>\max \{0, \operatorname{dim} M+\operatorname{dim} A-\operatorname{dim} \Sigma\},
$$

then the set of all $v \in V$, such that the map $F(v)$ is transverse to $A$, is residual and therefore dense in $V$. 
Let $(M, g)$ be a Riemannian manifold and let $\Sigma$ be a vector bundle over $M$ with a connection $\nabla$. Then the connection Laplacian

$$
\nabla^{*} \nabla: \quad C^{\infty}(\Sigma) \rightarrow C^{\infty}(\Sigma)
$$

is a linear elliptic differential operator of second order. In terms of a local $g$-orthonormal frame $\left(e_{i}\right)_{i=1}^{n}$ of $T M$ it is given by

$$
\nabla^{*} \nabla \psi=-\sum_{i=1}^{n} \nabla_{e_{i}} \nabla_{e_{i}} \psi+\sum_{i=1}^{n} \nabla_{\nabla_{e_{i}} e_{i}} \psi
$$

We will use the following unique continuation theorem due to Aronszajn ([6], quoted from [11]).

Theorem 2.4. Let $(M, g)$ be a connected Riemannian manifold and let $\Sigma$ be a vector bundle over $M$ with a connection $\nabla$. Let $P$ be an operator of the form $P=\nabla^{*} \nabla+P_{1}+P_{0}$ acting on sections of $\Sigma$, where $P_{1}, P_{0}$ are differential operators of order 1 and 0 respectively. Let $\psi$ be a solution to $P \psi=0$. If there exists a point, at which $\psi$ and all derivatives of $\psi$ of any order vanish, then $\psi$ vanishes identically.

If $(M, g, \Theta)$ is a closed Riemannian spin manifold, then from the connection $\nabla^{g}$ on the spinor bundle one obtains a connection Laplacian $\nabla^{*} \nabla$. Later we will apply the unique continuation theorem to the operator $P=$ $\left(D^{g}\right)^{2}-\lambda^{2}, \lambda \in \mathbb{R} \backslash\{0\}$. This is possible since for all $\psi \in C^{\infty}\left(\Sigma^{g} M\right)$ we have by the Schrödinger-Lichnerowicz formula

$$
\left(D^{g}\right)^{2} \psi=\nabla^{*} \nabla \psi+\frac{\mathrm{scal}^{g}}{4} \psi
$$

(see [21] p. 160), where scal ${ }^{g}$ is the scalar curvature of $(M, g)$.

\section{Green's function for the Dirac operator}

\subsection{The Bourguignon-Gauduchon trivialization}

Let $(M, g, \Theta)$ be a Riemannian spin manifold of dimension $n$. In this section we explain a local trivialization of the spinor bundle $\Sigma^{g} M$, which we will use in order to describe the expansion of Green's function for the Dirac operator. In the literature (e.g., [5]) it is known as the Bourguignon-Gauduchon trivialization. 
Let $p \in M$, let $U$ be an open neighborhood of $p$ in $M$ and let $V$ be an open neighborhood of 0 in $\mathbb{R}^{n}$, such that there exists a local parametrization $\rho: V \rightarrow U$ of $M$ by Riemannian normal coordinates with $\rho(0)=p$. The spinor bundle over the Euclidean space $\left(\mathbb{R}^{n}, g_{\text {eucl }}\right)$ with the unique spin structure will be denoted by $\Sigma \mathbb{R}^{n}$. The Clifford multiplications on $\Sigma^{g} M$ and on $\Sigma \mathbb{R}^{n}$ will both be denoted by a dot $\cdot$. It will be clear from the context which one of these two multiplications we mean.

Let $x \in V$. Then there exists $G_{x} \in \operatorname{End}\left(T_{x} V\right)$, such that for all vectors $v, w \in T_{x} V$ we have

$$
\left(\rho^{*} g\right)(v, w)=g_{\text {eucl }}\left(G_{x} v, w\right)
$$

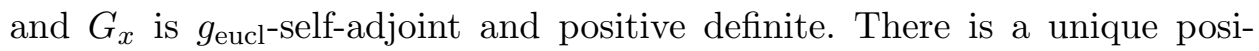
tive definite endomorphism $B_{x} \in \operatorname{End}\left(T_{x} V\right)$ such that we have $B_{x}^{2}=G_{x}^{-1}$.

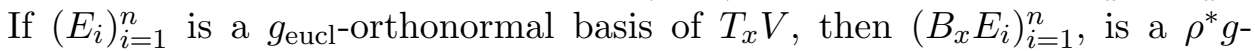
orthonormal basis of $T_{x} V$. Therefore the vectors $\left.d \rho\right|_{x} B_{x} E_{i}, 1 \leq i \leq n$, form a $g$-orthonormal basis of $T_{\rho(x)} M$. We assemble the maps $B_{x}$ to obtain a vector bundle endomorphism $B$ of $T V$ and we define

$$
b:\left.\quad T V \rightarrow T M\right|_{U}, \quad b=d \rho \circ B .
$$

From this we obtain an isomorphism of principal $\mathrm{SO}(n)$-bundles

$$
P_{\mathrm{SO}}\left(V, g_{\mathrm{eucl}}\right) \rightarrow P_{\mathrm{SO}}(U, g), \quad\left(E_{i}\right)_{i=1}^{n} \mapsto\left(b\left(E_{i}\right)\right)_{i=1}^{n},
$$

which lifts to an isomorphism of principal $\operatorname{Spin}(n)$-bundles

$$
c: \quad P_{\text {Spin }}\left(V, g_{\text {eucl }}\right) \rightarrow P_{\text {Spin }}(U, g) \text {. }
$$

We define

$$
\beta:\left.\left.\quad \Sigma \mathbb{R}^{n}\right|_{V} \rightarrow \Sigma^{g} M\right|_{U}, \quad[s, \sigma] \mapsto[c(s), \sigma]
$$

This gives an identification of the spinor bundles, which is a fibrewise isometry with respect to the bundle metrics on $\left.\Sigma \mathbb{R}^{n}\right|_{V}$ and on $\left.\Sigma^{g} M\right|_{U}$. Furthermore, for all $X \in T V$ and for all $\left.\varphi \in \Sigma \mathbb{R}^{n}\right|_{V}$ we have $\beta(X \cdot \varphi)=b(X) \cdot \beta(\varphi)$. We obtain an isomorphism

$$
A: \quad C^{\infty}\left(\left.\Sigma^{g} M\right|_{U}\right) \rightarrow C^{\infty}\left(\left.\Sigma \mathbb{R}^{n}\right|_{V}\right), \quad \psi \mapsto \beta^{-1} \circ \psi \circ \rho,
$$

which sends a spinor on $U$ to the corresponding spinor in the trivialization. Let $\nabla^{g}$ resp. $\nabla$ denote the Levi Civita connections on $(U, g)$ resp. on $\left(V, g_{\text {eucl }}\right)$ as well as their lifts to $\left.\Sigma^{g} M\right|_{U}$ and $\left.\Sigma \mathbb{R}^{n}\right|_{V}$. 
The connection $\nabla^{g}$ on $\left.\Sigma^{g} M\right|_{U}$ may be written as follows. Let $\left(e_{i}\right)_{i=1}^{n}$ be a positively oriented local orthonormal frame of $T M$ on $U$. There exists a locally defined section $s \in C^{\infty}\left(\left.\mathrm{P}_{\operatorname{Spin}}(M, g)\right|_{U}\right)$ such that $\left(e_{i}\right)_{i=1}^{n}=\Theta \circ s$ on $U$. Let $\left(E_{i}\right)_{i=1}^{N}$ be the standard basis of $\mathbb{C}^{N}$, where $N:=2^{[n / 2]}$. The section $s$ determines a local orthonormal frame $\left(\psi_{i}\right)_{i=1}^{N}$ of $\left.\Sigma^{g} M\right|_{U}$ via

$$
\psi_{i}=\left[s, E_{i}\right] \in C^{\infty}\left(\left.\Sigma^{g} M\right|_{U}\right), \quad i=1, \ldots, N
$$

We denote by $\partial$ the locally defined flat connection with respect to the local frame $\left(\psi_{i}\right)_{i=1}^{N}$, i.e., for $h_{1}, \ldots, h_{N} \in C^{\infty}(U, \mathbb{C})$ and $\left.X \in T M\right|_{U}$ we define

$$
\partial_{X}\left(\sum_{i=1}^{N} h_{i} \psi_{i}\right):=\sum_{i=1}^{N} X\left(h_{i}\right) \psi_{i}
$$

Then for all $\psi \in C^{\infty}\left(\left.\Sigma^{g} M\right|_{U}\right)$ we have

$$
\nabla_{e_{i}}^{g} \psi=\partial_{e_{i}} \psi+\frac{1}{4} \sum_{j, k=1}^{n} \widetilde{\Gamma}_{i j}^{k} e_{j} \cdot e_{k} \cdot \psi
$$

where

$$
\widetilde{\Gamma}_{i j}^{k}:=g\left(\nabla_{e_{i}}^{g} e_{j}, e_{k}\right)
$$

(see [21], p. 103, 110).

In particular, we can take the standard basis $\left(E_{i}\right)_{i=1}^{n}$ of $\mathbb{R}^{n}$ and put $e_{i}:=b\left(E_{i}\right), 1 \leq i \leq n$. We define the matrix coefficients $B_{i}^{j}$ by $B\left(E_{i}\right)=$ $\sum_{j=1}^{n} B_{i}^{j} E_{j}$. It follows that

$$
\begin{aligned}
A \nabla_{e_{i}}^{g} \psi & =\nabla_{d \rho^{-1}\left(e_{i}\right)} A \psi+\frac{1}{4} \sum_{j, k=1}^{n} \widetilde{\Gamma}_{i j}^{k} E_{j} \cdot E_{k} \cdot A \psi \\
& =\nabla_{B\left(E_{i}\right)} A \psi+\frac{1}{4} \sum_{j, k=1}^{n} \widetilde{\Gamma}_{i j}^{k} E_{j} \cdot E_{k} \cdot A \psi \\
& =\nabla_{E_{i}} A \psi+\sum_{j=1}^{n}\left(B_{i}^{j}-\delta_{i}^{j}\right) \nabla_{E_{j}} A \psi+\frac{1}{4} \sum_{j, k=1}^{n} \widetilde{\Gamma}_{i j}^{k} E_{j} \cdot E_{k} \cdot A \psi .
\end{aligned}
$$


Hence we obtain

$$
\begin{aligned}
A D^{g} \psi= & D^{g_{\mathrm{eucl}}} A \psi+\sum_{i, j=1}^{n}\left(B_{i}^{j}-\delta_{i}^{j}\right) E_{i} \cdot \nabla_{E_{j}} A \psi \\
& +\frac{1}{4} \sum_{i, j, k=1}^{n} \widetilde{\Gamma}_{i j}^{k} E_{i} \cdot E_{j} \cdot E_{k} \cdot A \psi
\end{aligned}
$$

Let $\partial_{j}:=d \rho\left(E_{j}\right), 1 \leq j \leq n$, be the coordinate vector fields of the normal coordinates. The Taylor expansion of the coefficient $g_{i j}$ of the metric around 0 is given by

$$
g_{i j}(x)=\delta_{i j}+\frac{1}{3} \sum_{a, b=1}^{n} R_{i a b j}(p) x_{a} x_{b}+O\left(|x|^{3}\right)
$$

where $R_{i a b j}=g\left(R\left(\partial_{b}, \partial_{j}\right) \partial_{a}, \partial_{i}\right)$ denotes the components of the Riemann curvature tensor (see e.g., [22], p. 42, 61). Since we have $\left(B_{i}^{j}\right)_{i j}=\left(g_{i j}\right)_{i j}^{-1 / 2}$ it follows that

$$
B_{i}^{j}(x)=\delta_{i}^{j}-\frac{1}{6} \sum_{a, b=1}^{n} R_{i a b j}(p) x_{a} x_{b}+O\left(|x|^{3}\right) .
$$

Since we have $\left.\nabla_{\partial_{k}}^{g} \partial_{r}\right|_{p}=0$ for all $k, r$, we obtain

$$
\widetilde{\Gamma}_{k r}^{m}(\rho(x))=O(|x|)
$$

as $x \rightarrow 0$ for all $m, k, r$.

\subsection{The Euclidean Dirac operator}

The aim of this section is to calculate preimages under the Dirac operator of certain spinors on $\mathbb{R}^{n} \backslash\{0\}$ with the Euclidean metric. The results will be useful for obtaining the expansion of Green's function for the Dirac operator on a closed spin manifold. 
Definition 3.1. For $k \in \mathbb{R}, m \in \mathbb{N}$ and $i \in\{0,1\}$ we define the vector subspaces $P_{k, m, i}\left(\mathbb{R}^{n}\right)$ of $C^{\infty}\left(\left.\Sigma \mathbb{R}^{n}\right|_{\mathbb{R}^{n} \backslash\{0\}}\right)$ as follows. For $k \neq 0$ define

$$
\begin{aligned}
& P_{k, m, 0}\left(\mathbb{R}^{n}\right):=\operatorname{span}\left\{\begin{array}{ll}
x \mapsto x_{i_{1}} \ldots x_{i_{m}}|x|^{k} \gamma & \begin{array}{l}
1 \leq i_{1}, \ldots, i_{m} \leq n, \\
\gamma \in \Sigma_{n} \text { constant }
\end{array}
\end{array}\right\} \\
& P_{k, m, 1}\left(\mathbb{R}^{n}\right):=\operatorname{span}\left\{\begin{array}{l|l}
x \mapsto x_{i_{1}} \ldots x_{i_{m}}|x|^{k} x \cdot \gamma \mid \begin{array}{l}
1 \leq i_{1}, \ldots, i_{m} \leq n, \\
\gamma \in \Sigma_{n} \text { constant }
\end{array}
\end{array}\right\}
\end{aligned}
$$

and furthermore define

$$
\begin{aligned}
& P_{0, m, 0}\left(\mathbb{R}^{n}\right):=\operatorname{span}\left\{\begin{array}{l|l}
x \mapsto x_{i_{1}} \ldots x_{i_{m}} \ln |x| \gamma \mid \begin{array}{l}
1 \leq i_{1}, \ldots, i_{m} \leq n, \\
\gamma \in \Sigma_{n} \text { constant }
\end{array}
\end{array}\right\} \\
& P_{0, m, 1}\left(\mathbb{R}^{n}\right):=\operatorname{span}\left\{\begin{array}{l|l}
x \mapsto x_{i_{1}} \ldots x_{i_{m}}(1-n \ln |x|) x \cdot \gamma \mid \begin{array}{l}
1 \leq i_{1}, \ldots, i_{m} \leq n, \\
\gamma \in \Sigma_{n} \text { constant }
\end{array}
\end{array}\right\} .
\end{aligned}
$$

With these definitions we can prove the following proposition.

Proposition 3.2. For all $m \in \mathbb{N}, k \in \mathbb{R}$ with $-n \leq k$ and $-n<k+m \leq 0$ we have

$$
P_{k, m, 0}\left(\mathbb{R}^{n}\right) \subset D^{g_{\mathrm{eucl}}}\left(\sum_{j=1}^{[(m+1) / 2]} P_{k+2 j, m+1-2 j, 0}\left(\mathbb{R}^{n}\right)+\sum_{j=0}^{[m / 2]} P_{k+2 j, m-2 j, 1}\left(\mathbb{R}^{n}\right)\right)
$$

For all $m \in \mathbb{N}, k \in \mathbb{R}$ with $-n \leq k$ and $-n<k+m+1 \leq 0$ we have

$$
P_{k, m, 1}\left(\mathbb{R}^{n}\right) \subset D^{g_{\text {eucl }}}\left(\sum_{j=0}^{[m / 2]} P_{k+2+2 j, m-2 j, 0}\left(\mathbb{R}^{n}\right)+\sum_{j=1}^{[(m+1) / 2]} P_{k+2 j, m+1-2 j, 1}\left(\mathbb{R}^{n}\right)\right) .
$$

Proof. We use induction on $m$. Let $m=0$ and let $\gamma$ be a constant spinor on $\left(\mathbb{R}^{n}, g_{\text {eucl }}\right)$. We want to prove that $P_{k, 0,0}\left(\mathbb{R}^{n}\right) \subset D^{g_{\text {eucl }}}\left(P_{k, 0,1}\left(\mathbb{R}^{n}\right)\right)$ for all $k$ with $-n<k \leq 0$ and $P_{k, 0,1}\left(\mathbb{R}^{n}\right) \subset D^{g_{\text {eucl }}}\left(P_{k+2,0,0}\left(\mathbb{R}^{n}\right)\right)$ for all $k$ with $-n \leq k$ and $k+1 \leq 0$. One calculates easily that

$$
\begin{aligned}
& D^{g_{\text {eucl }}}\left(-\frac{1}{n+k}|x|^{k} x \cdot \gamma\right)=|x|^{k} \gamma, \quad k \neq-n \\
& D^{g_{\text {eucl }}}\left(\frac{1-n \ln |x|}{n^{2}} x \cdot \gamma\right)=\ln |x| \gamma \text {, } \\
& D^{g_{\text {eucl }}}\left(\frac{1}{k+2}|x|^{k+2} \gamma\right)=|x|^{k} x \cdot \gamma, \quad k \neq-2 \\
& D^{g_{\text {eucl }}}(\ln |x| \gamma)=|x|^{-2} x \cdot \gamma .
\end{aligned}
$$


Using the definition of $P_{k, 0, i}\left(\mathbb{R}^{n}\right)$ one finds that the assertion holds for $m=0$.

Let $m \geq 1$ and assume that all the inclusions in the assertion hold for $m-1$. Using the equation $E_{i} \cdot x=-2 x_{i}-x \cdot E_{i}$ we find

$$
\begin{aligned}
& D^{g_{\text {eucl }}}\left(-x_{i_{1}} \ldots x_{i_{m}}|x|^{k} x \cdot \gamma\right) \\
& \quad=-\sum_{j=1}^{m} x_{i_{1}} \ldots \widehat{x_{i_{j}}} \ldots x_{i_{m}}|x|^{k} E_{i_{j}} \cdot x \cdot \gamma-x_{i_{1}} \ldots x_{i_{m}} D^{g_{\text {eucl }}}\left(|x|^{k} x \cdot \gamma\right) \\
& \quad=(2 m+n+k) x_{i_{1}} \ldots x_{i_{m}}|x|^{k} \gamma+\sum_{j=1}^{m} x_{i_{1}} \ldots \widehat{x_{i_{j}}} \ldots x_{i_{m}}|x|^{k} x \cdot E_{i_{j}} \cdot \gamma
\end{aligned}
$$

Since $E_{i_{j}} \cdot \gamma$ is a parallel spinor the sum on the right hand side is contained in $P_{k, m-1,1}\left(\mathbb{R}^{n}\right)$. We apply the induction hypothesis and since $2 m+n+k \neq 0$ we obtain the assertion for $P_{k, m, 0}\left(\mathbb{R}^{n}\right)$. We define $f_{k}(x):=\frac{1}{k}|x|^{k}$ for $k \neq 0$ and $f_{0}(x):=\ln |x|$. Then we find

$$
\begin{aligned}
& D^{g_{\text {eucl }}}\left(x_{i_{1}} \ldots x_{i_{m}} f_{k+2}(x) \gamma\right) \\
& \quad=x_{i_{1}} \ldots x_{i_{m}}|x|^{k} x \cdot \gamma+\sum_{j=1}^{m} x_{i_{1}} \ldots \widehat{x_{i_{j}}} \ldots x_{i_{m}} f_{k+2}(x) E_{i_{j}} \cdot \gamma
\end{aligned}
$$

The sum on the right hand side is in $P_{k+2, m-1,0}\left(\mathbb{R}^{n}\right)$. Again we apply the induction hypothesis and we obtain the assertion for $P_{k, m, 1}\left(\mathbb{R}^{n}\right)$.

\subsection{Expansion of Green's function}

Let $(M, g, \Theta)$ be a closed Riemannian spin manifold of dimension $n$ and let $\lambda \in \mathbb{R}$. Let $\pi_{i}: M \times M \rightarrow M, i=1,2$ be the projections. We define

$$
\Sigma^{g} M \otimes \Sigma^{g} M^{*}:=\pi_{1}^{*} \Sigma^{g} M \otimes\left(\pi_{2}^{*} \Sigma^{g} M\right)^{*}
$$

i.e., $\Sigma^{g} M \otimes \Sigma^{g} M^{*}$ is the vector bundle over $M \times M$ whose fiber over the point $(x, y) \in M \times M$ is given by $\operatorname{Hom}_{\mathbb{C}}\left(\Sigma_{y}^{g} M, \Sigma_{x}^{g} M\right)$. Let $\Delta:=\{(x, x) \mid x \in$ $M\}$ be the diagonal. In the following we will abbreviate

$$
\int_{M \backslash\{p\}}:=\lim _{\varepsilon \rightarrow 0} \int_{M \backslash B_{\varepsilon}(p)} .
$$

Definition 3.3. A smooth section $G_{\lambda}^{g}: M \times M \backslash \Delta \rightarrow \Sigma^{g} M \otimes \Sigma^{g} M^{*}$ which is locally integrable on $M \times M$ is called a Green's function for $D^{g}-\lambda$ if for all $p \in M$ and for all $\varphi \in \Sigma_{p}^{g} M$ the following holds: 
(1) For all $\psi \in \operatorname{im}\left(D^{g}-\lambda\right)$ we have

$$
\int_{M \backslash\{p\}}\left\langle\left(D^{g}-\lambda\right) \psi, G_{\lambda}^{g}(., p) \varphi\right\rangle \mathrm{dv}^{g}=\langle\psi(p), \varphi\rangle .
$$

(2) For all $\psi \in \operatorname{ker}\left(D^{g}-\lambda\right)$ we have

$$
\int_{M \backslash\{p\}}\left\langle\psi, G_{\lambda}^{g}(., p) \varphi\right\rangle \mathrm{dv}^{g}=0 .
$$

The smooth spinor $G_{\lambda}^{g}(., p) \varphi$ on $M \backslash\{p\}$ will sometimes also be called Green's function for $\varphi$. Let $P: C^{\infty}\left(\Sigma^{g} M\right) \rightarrow \operatorname{ker}\left(D^{g}-\lambda\right)$ denote the $L^{2}$-orthogonal projection. Then we have for all $\psi \in C^{\infty}\left(\Sigma^{g} M\right)$ and for all $\varphi \in \Sigma_{p}^{g} M$

$$
\int_{M \backslash\{p\}}\left\langle\left(D^{g}-\lambda\right) \psi, G_{\lambda}^{g}(., p) \varphi\right\rangle \mathrm{dv}^{g}=\langle\psi(p)-P \psi(p), \varphi\rangle .
$$

On Euclidean space we define a Green's function as follows.

Definition 3.4. Let $(M, g)=\left(\mathbb{R}^{n}, g_{\mathrm{eucl}}\right)$ with the unique spin structure. $A$ smooth section $G_{\lambda}^{g}: M \times M \backslash \Delta \rightarrow \Sigma^{g} M \otimes \Sigma^{g} M^{*}$ which is locally integrable on $M \times M$ is called a Green's function for $D^{g}-\lambda$ if for all $p \in M$, for all $\varphi \in \Sigma_{p}^{g} M$ and for all $\psi \in C^{\infty}\left(\Sigma^{g} M\right)$ with compact support the equation (3.5) holds.

Of course a Green's function for $D^{g_{\text {eucl }}}-\lambda$ is not uniquely determined by this definition. We will explicitly write down a Green's function for $D^{g_{\text {eucl }}}-$ $\lambda$. First observe that for every spinor $\chi \in C^{\infty}\left(\left.\Sigma \mathbb{R}^{n}\right|_{\mathbb{R}^{n} \backslash\{0\}}\right)$ and for every $\lambda \in \mathbb{R}$ the equation

$$
\left(D^{g_{\mathrm{eucl}}}-\lambda\right)\left(D^{g_{\mathrm{eucl}}}+\lambda\right) \chi=-\sum_{i=1}^{n} \nabla_{E_{i}} \nabla_{E_{i}} \chi-\lambda^{2} \chi
$$

holds on $\mathbb{R}^{n} \backslash\{0\}$. Let $\gamma$ be a constant spinor on $\left(\mathbb{R}^{n}, g_{\text {eucl }}\right)$ and let $g$ be a solution to the ordinary differential equation

$$
g^{\prime \prime}(z)+\frac{n-1}{z} g^{\prime}(z)+\lambda^{2} g(z)=-\delta_{0}
$$

which is smooth on $(0, \infty)$. If we define $f \in C^{\infty}\left(\mathbb{R}^{n} \backslash\{0\}, \mathbb{R}\right)$ by $f(x):=$ $g(|x|)$, then the spinor $G_{\lambda}^{g_{\text {eucl }}}(., 0) \gamma:=\left(D^{g_{\text {eucl }}}+\lambda\right)(f \gamma)$ is a Green's function. 
In the following let $\Gamma$ denote the Gamma function and $J_{m}, Y_{m}$ the Bessel functions of the first and second kind for the parameter $m \in \mathbb{R}$. In the notation of [7], p. 360 they are defined for $z \in(0, \infty)$ by

$$
\begin{aligned}
& J_{m}(z)=\frac{1}{2^{m} \Gamma(m+1)} z^{m}\left(1+\sum_{k=1}^{\infty} a_{k} z^{2 k}\right), \quad m \in \mathbb{R}, \\
& Y_{0}(z)=\frac{2}{\pi}\left(\ln \left(\frac{z}{2}\right)+c\right) J_{0}(z)+\sum_{k=1}^{\infty} b_{k} z^{2 k}, \\
& Y_{m}(z)=-\frac{2^{m}}{\pi} \Gamma(m) z^{-m}\left(1+\sum_{k=1}^{\infty} c_{k} z^{2 k}\right), \quad m=\frac{1}{2}+k, k \in \mathbb{N}, \\
& Y_{m}(z)=-\frac{2^{m}}{\pi} \Gamma(m) z^{-m}\left(1+\sum_{k=1}^{\infty} d_{k} z^{2 k}\right)+\frac{2}{\pi} \ln \left(\frac{z}{2}\right) J_{m}(z), \quad m \in \mathbb{N} \backslash\{0\},
\end{aligned}
$$

where $c$ is a real constant, the $a_{k}, b_{k}, c_{k}, d_{k}$ are real coefficients, the $a_{k}$, $c_{k}, d_{k}$ depend on $m$ and all the power series converge for all $z \in(0, \infty)$. Let $\omega_{n-1}=\operatorname{vol}\left(S^{n-1}, g_{\text {can }}\right)$ be the volume of the $(n-1)$-dimensional unit sphere with the standard metric.

Theorem 3.5. Let $m:=\frac{n-2}{2}$. We define $f_{\lambda}: \mathbb{R}^{n} \backslash\{0\} \rightarrow \mathbb{R}$ as follows. For $\lambda \neq 0$ and $n=2$

$$
f_{\lambda}(x):=-\frac{1}{4} Y_{0}(|\lambda x|)+\frac{\ln |\lambda|-\ln (2)+c}{2 \pi} J_{0}(|\lambda x|),
$$

for $\lambda \neq 0$ and odd $n \geq 3$

$$
f_{\lambda}(x):=-\frac{\pi|\lambda|^{m}}{2^{m} \Gamma(m)(n-2) \omega_{n-1}}|x|^{-m} Y_{m}(|\lambda x|),
$$

for $\lambda \neq 0$ and even $n \geq 4$

$f_{\lambda}(x):=-\frac{\pi|\lambda|^{m}}{2^{m} \Gamma(m)(n-2) \omega_{n-1}}|x|^{-m}\left(Y_{m}(|\lambda x|)-\frac{2(\ln |\lambda|-\ln (2))}{\pi} J_{m}(|\lambda x|)\right)$

and

$$
f_{0}(x):=-\frac{1}{2 \pi} \ln |x|, \quad n=2, \quad f_{0}(x):=\frac{1}{(n-2) \omega_{n-1}|x|^{n-2}}, \quad n \geq 3 .
$$

Then for every constant spinor $\gamma$ on $\mathbb{R}^{n}$ a Green's function for $D^{g_{\mathrm{eucl}}}-\lambda$ is given by

$$
G_{\lambda}^{g_{\text {eucl }}}(x, 0) \gamma=\left(D^{g_{\text {eucl }}}+\lambda\right)\left(f_{\lambda} \gamma\right)(x)
$$


Corollary 3.6. For every constant spinor $\gamma \in \Sigma_{n}$ there exists a Green's function of $D^{g_{\text {eucl }}}-\lambda$, which has the following form. For $n=2$

$$
G_{\lambda}^{g_{\text {eucl }}}(x, 0) \gamma=-\frac{1}{2 \pi|x|} \frac{x}{|x|} \cdot \gamma-\frac{\lambda}{2 \pi} \ln |x| \gamma+\ln |x| \vartheta_{\lambda}(x)+\zeta_{\lambda}(x),
$$

for odd $n \geq 3$

$$
G_{\lambda}^{g_{\text {eucl }}}(x, 0) \gamma=-\frac{1}{\omega_{n-1}|x|^{n-1}} \frac{x}{|x|} \cdot \gamma+\frac{\lambda}{(n-2) \omega_{n-1}|x|^{n-2}} \gamma+|x|^{2-n} \zeta_{\lambda}(x),
$$

for even $n \geq 4$

$$
\begin{aligned}
G_{\lambda}^{g_{\text {eucl }}}(x, 0) \gamma= & -\frac{1}{\omega_{n-1}|x|^{n-1}} \frac{x}{|x|} \cdot \gamma+\frac{\lambda}{(n-2) \omega_{n-1}|x|^{n-2}} \gamma+|x|^{2-n} \zeta_{\lambda}(x) \\
& -\frac{\lambda^{n-1}}{2^{n-2} \Gamma\left(\frac{n}{2}\right)^{2} \omega_{n-1}} \ln |x| \gamma+\ln |x| \vartheta_{\lambda}(x)
\end{aligned}
$$

where for every $n$ and for every $\lambda$ the spinors $\vartheta_{\lambda}, \zeta_{\lambda}$ extend smoothly to $\mathbb{R}^{n}$ and satisfy

$$
\left|\zeta_{\lambda}(x)\right|_{g_{\text {eucl }}}=O(|x|), \quad\left|\vartheta_{\lambda}(x)\right|_{g_{\text {eucl }}}=O(|x|) \quad \text { as } x \rightarrow 0
$$

and where for every $n$ and for every $x$ the spinors $\vartheta_{\lambda}(x), \zeta_{\lambda}(x) \in \Sigma_{n}$ are power series in $\lambda$ with $\vartheta_{0}(x)=\zeta_{0}(x)=0$.

Proof of Corollary 3.6. Using the definition of $f_{\lambda}$ we find for $n=2$

$$
f_{\lambda}(x)=-\frac{1}{2 \pi} \ln |x|\left(1+\sum_{k=1}^{\infty} a_{k}|\lambda x|^{2 k}\right)-\frac{1}{4} \sum_{k=1}^{\infty} b_{k}|\lambda x|^{2 k},
$$

for odd $n \geq 3$

$$
f_{\lambda}(x)=\frac{1}{(n-2) \omega_{n-1}|x|^{n-2}}\left(1+\sum_{k=1}^{\infty} c_{k}|\lambda x|^{2 k}\right)
$$

and for even $n \geq 4$

$$
\begin{aligned}
f_{\lambda}(x)= & \frac{1}{(n-2) \omega_{n-1}|x|^{n-2}}\left(1+\sum_{k=1}^{\infty} d_{k}|\lambda x|^{2 k}\right) \\
& -\frac{\lambda^{n-2}}{2^{n-2}(m !)^{2} \omega_{n-1}} \ln |x|\left(1+\sum_{k=1}^{\infty} a_{k}|\lambda x|^{2 k}\right) .
\end{aligned}
$$


The assertion follows.

Proof of Theorem 3.5. Let $f_{\lambda}$ be as in the assertion and write $f_{\lambda}(x)=g_{\lambda}(|x|)$ with $g_{\lambda}:(0, \infty) \rightarrow \mathbb{R}$. Then $g_{\lambda}$ solves the Equation (3.8). It remains to show that $\left(D^{g_{\text {eucl }}}+\lambda\right)\left(f_{\lambda} \gamma\right)$ satisfies $(3.5)$. The calculation in the proof of Corollary 3.6 shows

$$
\left(D^{g_{\mathrm{eucl}}}+\lambda\right)\left(f_{\lambda} \gamma\right)(x)=-\frac{1}{\omega_{n-1}} \frac{x}{|x|^{n}} \cdot \gamma+\zeta(x),
$$

where $|\zeta(x)|_{g_{\text {eucl }}}=o\left(|x|^{1-n}\right)$ as $x \rightarrow 0$. For any Riemannian spin manifold $(M, g, \Theta)$ with boundary $\partial M$ and $\psi, \varphi$ compactly supported spinors we have

$$
\left(D^{g} \psi, \varphi\right)_{2}-\left(\psi, D^{g} \varphi\right)_{2}=\int_{\partial M}\langle\nu \cdot \psi, \varphi\rangle d A,
$$

where $\nu$ is the outer unit normal vector field on $\partial M$ (see [21], p. 115). We apply this equation to $\left(\mathbb{R}^{n} \backslash B_{\varepsilon}(0), g_{\text {eucl }}\right)$ and $\nu(x):=-\frac{x}{|x|}$ and we obtain the assertion.

Definition 3.7. For $m \in \mathbb{R}$ we define

$$
P_{m}\left(\mathbb{R}^{n}\right):=\sum_{\substack{r+s+t \geq m \\ r \geq-n}} P_{r, s, t}\left(\mathbb{R}^{n}\right)+\left(C^{\infty}\left(\left.\Sigma \mathbb{R}^{n}\right|_{\mathbb{R}^{n} \backslash\{0\}}\right) \cap C^{0}\left(\Sigma \mathbb{R}^{n}\right)\right),
$$

where the second space on the right hand side is the space of all spinors which are smooth on $\mathbb{R}^{n} \backslash\{0\}$ and have a continuous extension to $\mathbb{R}^{n}$.

Remark 3.8. Let $\vartheta \in P_{m}\left(\mathbb{R}^{n}\right)$. Then we have $E_{i} \cdot \vartheta \in P_{m}\left(\mathbb{R}^{n}\right)$ for all $i \in$ $\{1, \ldots, n\}$. If $f \in C^{\infty}\left(\mathbb{R}^{n}, \mathbb{C}\right)$ then from Taylor's formula for $f$ it follows that the spinor $f \vartheta$ is in $P_{m}\left(\mathbb{R}^{n}\right)$.

Remark 3.9. If $m>0$ then every spinor in $P_{m}\left(\mathbb{R}^{n}\right)$ has a continuous extension to $\mathbb{R}^{n}$. Furthermore, by Proposition 3.2 it follows that for all $m \in(-n, 0]$ we have

$$
\begin{aligned}
P_{m}\left(\mathbb{R}^{n}\right) & =\sum_{\substack{r+s+t=m \\
r \geq-n}} P_{r, s, t}\left(\mathbb{R}^{n}\right)+P_{m+1}\left(\mathbb{R}^{n}\right) \\
& \subset D^{g_{\text {eucl }}}\left(P_{m+1}\left(\mathbb{R}^{n}\right)\right)+P_{m+1}\left(\mathbb{R}^{n}\right) .
\end{aligned}
$$

Lemma 3.10. Let $(M, g, \Theta)$ be a closed Riemannian spin manifold of dimension $n$ and let $\lambda \in \mathbb{R}$. Let $\gamma \in \Sigma_{n}$ be a constant spinor on $\mathbb{R}^{n}$. Then the spinor 
$G_{\lambda}^{g_{\text {eucl }}}(., 0) \gamma$ is in $P_{1-n}\left(\mathbb{R}^{n}\right)$. Let the matrix coefficients $B_{i}^{j}$ be defined as in (3.2). Then for all $i$ the spinor

$$
x \mapsto \sum_{j=1}^{n}\left(B_{i}^{j}(x)-\delta_{i}^{j}\right) \nabla_{E_{j}} G_{\lambda}^{g_{\mathrm{eucl}}}(x, 0) \gamma
$$

is in $P_{2-n}\left(\mathbb{R}^{n}\right)$.

Proof. The assertion for $G_{\lambda}^{g_{\text {eucl }}}(., 0) \gamma$ can be seen immediately from Corollary 3.6. Let $f_{\lambda}$ be as in Theorem 3.5 and write $f_{\lambda}(x)=g_{\lambda}(|x|)$ with a function $g_{\lambda}:(0, \infty) \rightarrow \mathbb{R}$. Then we have

$$
G_{\lambda}^{g_{\text {eucl }}}(x, 0) \gamma=\frac{g_{\lambda}^{\prime}(|x|)}{|x|} x \cdot \gamma+\lambda g_{\lambda}(|x|) \gamma
$$

and for every $j \in\{1, \ldots, n\}$ we have

$$
\begin{aligned}
\nabla_{E_{j}} G_{\lambda}^{g_{\text {eucl }}(x, 0) \gamma=} & \frac{g_{\lambda}^{\prime \prime}(|x|) x_{j}}{|x|^{2}} x \cdot \gamma-\frac{g_{\lambda}^{\prime}(|x|) x_{j}}{|x|^{3}} x \cdot \gamma \\
& +\frac{g_{\lambda}^{\prime}(|x|)}{|x|} E_{j} \cdot \gamma+\lambda \frac{g_{\lambda}^{\prime}(|x|) x_{j}}{|x|} \gamma
\end{aligned}
$$

Since the exponential map is a radial isometry, we have $\sum_{j=1}^{n} g_{i j}(x) x_{j}=x_{i}$ and thus $\sum_{j=1}^{n} B_{i}^{j}(x) x_{j}=x_{i}$ for every fixed $i$. It follows that

$$
\sum_{j=1}^{n}\left(B_{i}^{j}(x)-\delta_{i}^{j}\right) \nabla_{E_{j}} G_{\lambda}^{g_{\text {eucl }}}(x, 0) \gamma=\sum_{j=1}^{n}\left(B_{i}^{j}(x)-\delta_{i}^{j}\right) \frac{g_{\lambda}^{\prime}(|x|)}{|x|} E_{j} \cdot \gamma .
$$

Since we have $g_{\lambda}^{\prime}(|x|)=O\left(|x|^{1-n}\right)$ as $x \rightarrow 0$ the assertion now follows from the Taylor expansion (3.3) of $B_{i}^{j}(x)$.

Next we prove existence and uniqueness of Green's function for $D^{g}-\lambda$ on a closed Riemannian spin manifold in such a way that we also obtain the expansion of Green's function around the singularity. The idea is to apply the equation (3.2) for the Dirac operator in the trivialization to a Euclidean Green's function and then determine the correction terms. For $\lambda=0$ this has been carried out in [5], where for some technical steps Sobolev embeddings were used. We present a more simple argument using the preimages under the Dirac operator from Proposition 3.2.

In the following for a fixed point $p \in M$ let $\rho: V \rightarrow U$ be a local parametrization of $M$ by Riemannian normal coordinates, where $U \subset M$ 
is an open neighborhood of $p, V \subset \mathbb{R}^{n}$ is an open neighborhood of 0 and $\rho(0)=p$. Furthermore let

$$
\beta:\left.\left.\quad \Sigma \mathbb{R}^{n}\right|_{V} \rightarrow \Sigma^{g} M\right|_{U}, \quad A: \quad C^{\infty}\left(\left.\Sigma^{g} M\right|_{U}\right) \rightarrow C^{\infty}\left(\left.\Sigma \mathbb{R}^{n}\right|_{V}\right)
$$

denote the maps which send a spinor to its corresponding spinor in the Bourguignon-Gauduchon trivialization defined in Section 3.1.

Theorem 3.11. Let $(M, g, \Theta)$ be a closed $n$-dimensional Riemannian spin manifold, $p \in M$. For every $\varphi \in \Sigma_{p}^{g} M$ there exists a unique Green's function $G_{\lambda}^{g}(., p) \varphi$. If $\gamma:=\beta^{-1} \varphi \in \Sigma_{n}$ is the constant spinor on $\mathbb{R}^{n}$ corresponding to $\varphi$, then the first two terms of the expansion of $A G_{\lambda}^{g}(., p) \varphi$ at 0 coincide with the first two terms of the expansion of $G_{\lambda}^{g_{\text {eucl }}}(., 0) \gamma$ given in Corollary 3.6.

Proof. Let $\varepsilon>0$ such that $B_{2 \varepsilon}(0) \subset V$ and let $\eta: \mathbb{R}^{n} \rightarrow[0,1]$ be a smooth function with $\operatorname{supp}(\eta) \subset B_{2 \varepsilon}(0)$ and $\eta \equiv 1$ on $B_{\varepsilon}(0)$. Then the spinor $\Theta_{1}$ defined on $\mathbb{R}^{n} \backslash\{0\}$ by $\Theta_{1}(x):=\eta(x) G_{\lambda}^{g_{\text {eucl }}}(x, 0) \gamma$ is smooth on $\mathbb{R}^{n} \backslash\{0\}$. For $r \in\{1, \ldots, n\}$ we define smooth spinors $\Phi_{r}$ on $M \backslash\{p\}$ and $\Theta_{r+1}$ on $\mathbb{R}^{n} \backslash\{0\}$ inductively as follows. For $r=1$ we define

$$
\Phi_{1}(q):= \begin{cases}A^{-1} \Theta_{1}(q), & q \in U \backslash\{p\} \\ 0, & q \in M \backslash U\end{cases}
$$

and

$$
\Theta_{2}(x):=\left\{\begin{array}{ll}
A\left(D^{g}-\lambda\right) \Phi_{1}(x), & x \in V \backslash\{0\} \\
0, & x \in \mathbb{R}^{n} \backslash V
\end{array} .\right.
$$

By the formula (3.2) for the Dirac operator in the trivialization we have on $V \backslash\{0\}$

$$
\begin{aligned}
\Theta_{2}= & \left(D^{g_{\text {eucl }}}-\lambda\right) \Theta_{1}+\sum_{i, j=1}^{n}\left(B_{i}^{j}-\delta_{i}^{j}\right) E_{i} \cdot \nabla_{E_{j}} \Theta_{1} \\
& +\frac{1}{4} \sum_{i, j, k=1}^{n} \widetilde{\Gamma}_{i j}^{k} E_{i} \cdot E_{j} \cdot E_{k} \cdot \Theta_{1} .
\end{aligned}
$$

The first term vanishes on $B_{\varepsilon}(0) \backslash\{0\}$. It follows from the expansions of $\widetilde{\Gamma}_{i j}^{k}$ and $B_{i}^{j}-\delta_{i}^{j}$ in (3.3), (3.4) and from Lemma 3.10 that $\Theta_{2} \in P_{2-n}\left(\mathbb{R}^{n}\right)$.

Next let $r \in\{2, \ldots, n\}$ and assume that $\Phi_{r-1}$ and $\Theta_{r}$ have already been defined. We may assume that $\Theta_{r} \in P_{r-n}\left(\mathbb{R}^{n}\right)$. By Remark 3.9 there exists 
$\beta_{r+1} \in P_{r+1-n}\left(\mathbb{R}^{n}\right) \quad$ such that $\quad \Theta_{r}-\left(D^{g_{\text {eucl }}}-\lambda\right) \beta_{r+1} \in P_{r+1-n}\left(\mathbb{R}^{n}\right)$.

We define $\Phi_{r}$ and $\Theta_{r+1}$ by

$$
\Phi_{r}(q):= \begin{cases}\Phi_{r-1}(q)-A^{-1}\left(\eta \beta_{r+1}\right)(q), & q \in U \backslash\{p\} \\ 0, & q \in M \backslash U\end{cases}
$$

and

$$
\Theta_{r+1}(x):=\left\{\begin{array}{ll}
A\left(D^{g}-\lambda\right) \Phi_{r}(x), & x \in V \backslash\{0\} \\
0, & x \in \mathbb{R}^{n} \backslash V
\end{array} .\right.
$$

By the formula (3.2) for the Dirac operator in the trivialization we have on $B_{\varepsilon}(0) \backslash\{0\}$

$$
\begin{aligned}
\Theta_{r+1}= & A\left(D^{g}-\lambda\right) \Phi_{r-1}-A\left(D^{g}-\lambda\right) A^{-1} \beta_{r+1} \\
= & \Theta_{r}-\left(D^{g_{\text {eucl }}}-\lambda\right) \beta_{r+1}-\sum_{i, j=1}^{n}\left(B_{i}^{j}-\delta_{i}^{j}\right) E_{i} \cdot \nabla_{E_{j}} \beta_{r+1} \\
& -\frac{1}{4} \sum_{i, j, k=1}^{n} \widetilde{\Gamma}_{i j}^{k} E_{i} \cdot E_{j} \cdot E_{k} \cdot \beta_{r+1} .
\end{aligned}
$$

Using the expansions of $\widetilde{\Gamma}_{i j}^{k}$ and $B_{i}^{j}-\delta_{i}^{j}$ in (3.3) and (3.4) we conclude that $\Theta_{r+1} \in P_{r+1-n}\left(\mathbb{R}^{n}\right)$.

We see that $\Theta_{n+1}$ has a continuous extension to $\mathbb{R}^{n}$ and we obtain a continuous extension $\Psi$ of $\left(D^{g}-\lambda\right) \Phi_{n}$ to all of $M$. Thus there exists

$$
\Psi^{\prime} \in C^{\infty}\left(\left.\Sigma^{g} M\right|_{M \backslash\{p\}}\right) \cap H^{1}\left(\Sigma^{g} M\right)
$$

such that $\left(D^{g}-\lambda\right) \Psi^{\prime}=P \Psi-\Psi$. Define

$$
\Gamma:=\Phi_{n}+\Psi^{\prime}, \quad \Theta:=-\eta \beta_{3}-\cdots-\eta \beta_{n+1}+A \Psi^{\prime} .
$$

Then on $B_{\varepsilon}(0) \backslash\{0\}$ we have $A \Gamma=G_{\lambda}^{g_{\text {eucl }}}(., 0) \gamma+\Theta$.

If $\psi_{1}, \ldots, \psi_{d}$ is an $L^{2}$-orthonormal basis of $\operatorname{ker}\left(D^{g}-\lambda\right)$, then for every $i \in\{1, \ldots, d\}$ the integral

$$
C_{i}:=\int_{M \backslash\{p\}}\left\langle\Gamma, \psi_{i}\right\rangle \mathrm{dv}^{g}
$$

exists, since we have $|A \Gamma(x)|_{g_{\text {eucl }}}=O\left(|x|^{1-n}\right)$ as $x \rightarrow 0$. Then

$$
G_{\lambda}^{g}(., p) \varphi:=\Gamma-\sum_{i=1}^{d} C_{i} \psi_{i}
$$


satisfies (3.5) and (3.6) and thus is a Green's function. Uniqueness also follows from (3.5) and (3.6). The statement on the expansion of $A G_{\lambda}^{g}(., p) \varphi$ is obvious, since we have $\Theta \in P_{3-n}\left(\mathbb{R}^{n}\right)$.

\section{Zero sets of eigenspinors}

\subsection{Eigenspinors in dimensions 2 and 3}

In this section, we prove Theorem 1.1. Assume that $n \in\{2,3\}$. Then there exists a quaternionic structure on the spinor bundle, i.e., a conjugate linear endomorphism $J$ of $\Sigma^{g} M$, which satisfies $J^{2}=-\mathrm{Id}$. Furthermore, $J$ is parallel and commutes with Clifford multiplication (see e.g., [15], p. 33). It follows that $J$ commutes with the Dirac operator and thus every eigenspace of $D^{g}$ has even complex dimension. Therefore, the following notation introduced by Dahl (see [13]) is useful.

Definition 4.1. Let $n \in\{2,3\}$. An eigenvalue $\lambda$ of $D^{g}$ is called simple, if one has $\operatorname{dim}_{\mathbb{C}} \operatorname{ker}\left(D^{g}-\lambda\right)=2$.

If $\lambda$ is a simple eigenvalue of $D^{g}$, then one can choose an $L^{2}$-orthonormal basis of $\operatorname{ker}\left(D^{g}-\lambda\right)$ of the form $\{\psi, J \psi\}$.

For every $g \in R(M)$ we enumerate the non-zero eigenvalues of $D^{g}$ in the following way

$$
\cdots \leq \lambda_{-2}(g) \leq \lambda_{-1}(g)<0<\lambda_{1}(g) \leq \lambda_{2}(g) \leq \cdots
$$

Here all the non-zero eigenvalues are repeated by half of their complex multiplicities, while $\operatorname{dim} \operatorname{ker}\left(D^{g}\right) \geq 0$ is arbitrary. For $m \in \mathbb{N} \backslash\{0\}$ we define

$$
\begin{aligned}
& S_{m}(M):=\left\{g \in R(M) \mid \lambda_{-m}(g), \ldots, \lambda_{m}(g) \text { are simple }\right\} \\
& N_{m}(M):=\left\{g \in R(M) \mid \begin{array}{l}
\text { all eigenspinors to } \lambda_{-m}(g), \ldots, \lambda_{m}(g) \\
\text { are nowhere zero }
\end{array}\right\} .
\end{aligned}
$$

Remark 4.2. Let $(M, \Theta)$ be a closed spin manifold of dimension $n \in\{2,3\}$. It is known that there exists a subset $S_{0}(M, \Theta) \subset R(M)$ which is open and dense in $R(M)$ such that the map

$$
S_{0}(M, \Theta) \rightarrow \mathbb{N}, \quad g \mapsto \operatorname{dim}_{\mathbb{C}} \operatorname{ker}\left(D^{g}\right)
$$

is constant. Furthermore, for $n=3$ this constant is 0 while for $n=2$ it is either 0 or 2 depending on the topology of $M$ and on the spin structure $\Theta$ 
(see [4, 23]). Dahl has shown that for $n \in\{2,3\}$ and for every $m \in \mathbb{N} \backslash\{0\}$ the subset $S_{0}(M, \Theta) \cap S_{m}(M)$ is open and dense in $R(M)$. His proof shows that for every $g \in R(M)$ and for every $m \in \mathbb{N} \backslash\{0\}$ the subset $S_{m}(M) \cap[g]$ is open and dense in $[g]$. All the statements in this remark hold with respect to all $C^{k}$-topologies, $k \geq 1$, on $R(M)$.

Since for $n=3$ for all $g \in S_{0}(M, \Theta)$ we have $\operatorname{dim}_{\mathbb{C}} \operatorname{ker}\left(D^{g}\right)=0$, it is sufficient for the proof of Theorem 1.1 in both cases $n \in\{2,3\}$ to consider non-harmonic eigenspinors. More precisely it is sufficient to prove the following theorem.

Theorem 4.3. Let $M$ be a closed connected spin manifold of dimension 2 or 3 and let $m \in \mathbb{N} \backslash\{0\}$. Then $S_{0}(M, \Theta) \cap N_{m}(M)$ is open and dense in $R(M)$. Furthermore, for every $g \in R(M)$ the subset $N_{m}(M) \cap[g]$ is open and dense in $[g]$.

In order to show density we will use Theorem 2.3 for families of spinors parametrized by Riemannian metrics. At first one might want to use the infinite-dimensional version of Theorem 2.3, where $V$ is equal to the space of all $C^{k}$-metrics on $M$ for some fixed $k \geq 1$ (see [24]). However, for non-smooth metrics $g$ the coefficients of the Dirac operator $D^{g}$ in a local chart and a local trivialization are not smooth by (3.2) and thus we cannot expect that the eigenspinors are smooth and that we can apply Theorem 2.4. Therefore we will use Theorem 2.3 with $V$ equal to a finite-dimensional manifold contained in $[g]$ of the form

$$
V_{f_{1} \ldots f_{s}}:=\left\{\left(1+\sum_{i=1}^{s} t_{i} f_{i}\right) g \mid t_{1}, \ldots, t_{s} \in \mathbb{R}\right\} \cap R(M),
$$

where $s \in \mathbb{N} \backslash\{0\}$ and $f_{1}, \ldots, f_{s} \in C^{\infty}(M, \mathbb{R})$.

Our first aim is to construct a map, which assigns to a Riemannian metric $h$ an eigenspinor of $D^{g, h}$ in a continuous way.

Lemma 4.4. Let $m \in \mathbb{N} \backslash\{0\}$ and let $g \in S_{0}(M, \Theta) \cap S_{m}(M)$. There exists an open neighborhood $V \subset R(M)$ of $g$ such that for every $i \in\{-m, \ldots, m\} \backslash$ $\{0\}$ and for every $L^{2}$-normalized eigenspinor $\psi$ of $D^{g}$ corresponding to $\lambda_{i}(g)$ there exists a map $F_{\psi}: V \rightarrow C^{\infty}\left(\Sigma^{g} M\right)$ with the following properties:

(1) For every $h \in V$ the spinor $F_{\psi}(h)$ is an eigenspinor of $D^{g, h}$ corresponding to $\lambda_{i}(h)$. 
(2) The map $F_{\psi}^{\mathrm{ev}}: V \times M \rightarrow \Sigma^{g} M$ defined by $F_{\psi}^{\mathrm{ev}}(h, x):=F_{\psi}(h)(x)$ is continuous.

(3) For all functions $f_{1}, \ldots, f_{s} \in C^{\infty}(M, \mathbb{R})$ the restriction of the map $F_{\psi}^{\mathrm{ev}}$ to $\left(V_{f_{1} \ldots f_{s}} \cap V\right) \times M$ is a $C^{1}$-map.

Proof. Let $h \in R(M)$. For every $t \in[0,1]$ the tensor field $g_{t}:=g+t(h-g)$ is a Riemannian metric on $M$. Then by Lemma 2.1 there exist real analytic functions

$$
[0,1] \ni t \mapsto \lambda_{i, t}^{h} \in \mathbb{R}, \quad i \in\{-m, \ldots, m\} \backslash\{0\},
$$

such that for every $i \in\{-m, \ldots, m\} \backslash\{0\}$ and for every $t \in[0,1]$ the number $\lambda_{i, t}^{h}$ is an eigenvalue of $D^{g, g_{t}}$ and such that for every $i$ we have $\lambda_{i, 0}^{h}=\lambda_{i}(g)$. There exists an open neighborhood $V$ of $g$ which is contained in $S_{0}(M, \Theta)$ such that for every $h \in V$, for every $i \in\{-m, \ldots, m\} \backslash\{0\}$ and for every $t \in[0,1]$ we have

$$
\lambda_{i, t}^{h}=\lambda_{i}\left(g_{t}\right)
$$

i.e., the eigenvalue functions are nowhere zero on $[0,1]$ and their graphs do not intersect. This follows from the continuity of the eigenvalues of the Dirac operator in the $C^{1}$-topology (see e.g., Proposition 7.1 in [10]) together with the fact that for every $h \in S_{0}(M, \Theta)$ we have $\operatorname{dim} \operatorname{ker}\left(D^{h}\right)=\operatorname{dim} \operatorname{ker}\left(D^{g}\right)$.

Next let $h \in V$, let $i \in\{-m, \ldots, m\} \backslash\{0\}$ and let $\psi$ be an eigenspinor of $D^{g}$ corresponding to $\lambda:=\lambda_{i}(g)$. By Lemma 2.1 there exists a real analytic family $t \mapsto \lambda_{t}, t \in[0,1]$, of eigenvalues of $D^{g, g_{t}}$ such that $\lambda_{0}=\lambda$ and there exists a real analytic family $t \mapsto \chi_{t} \in C^{\infty}\left(\Sigma^{g} M\right), t \in[0,1]$, of $L^{2}$-normalized eigenspinors of $D^{g, g_{t}}$ corresponding to $\lambda_{t}$. If $t \mapsto \zeta_{t}, t \in[0,1]$, is another such family of eigenspinors, then there exist analytic functions $a, b:[0,1] \rightarrow \mathbb{C}$ such that for all $t \in[0,1]$ we have $\zeta_{t}=a(t) \chi_{t}+b(t) J \chi_{t}$ and $|a(t)|^{2}+|b(t)|^{2}=1$. In order to fix a family of eigenspinors we apply a unitary transformation to $\chi_{t}$ such that for every $t$ small enough its component in $\operatorname{ker}\left(D^{g}-\lambda\right)$ becomes a positive multiple of $\psi$. More precisely for every $t \in[0,1]$ we define $f(t):=$ $\left(\chi_{t}, \psi\right)_{L^{2}}$ and $g(t):=\left(\chi_{t}, J \psi\right)_{L^{2}}$ and for every $t \in[0,1]$ such that $|f(t)|^{2}+$ $|g(t)|^{2}>0$ we define

$$
\psi_{t}:=\frac{\overline{f(t)} \chi_{t}-g(t) J \chi_{t}}{\left(|f(t)|^{2}+|g(t)|^{2}\right)^{1 / 2}}
$$

Then for all $t \in[0,1]$ such that $|f(t)|^{2}+|g(t)|^{2}>0$ the spinor $\psi_{t}$ is an eigenspinor of $D^{g, g_{t}}$ corresponding to $\lambda_{t}$ and we have $\psi_{0}=\psi$. By this definition 
we have fixed a family of eigenspinors. After possibly shrinking $V$ we may assume that for all metrics $h \in V$ the family $t \mapsto \psi_{t}$ is defined for all $t \in[0,1]$. We define $F_{\psi}(h):=\psi_{1}$. By (4.1) this eigenspinor of $D^{g, h}$ corresponds to $\lambda_{i}(h)$. If $\xi \in \operatorname{ker}\left(D^{g}-\lambda\right)$ is another $L^{2}$-normalized eigenspinor then there exist $c, d \in \mathbb{C}$ such that $|c|^{2}+|d|^{2}=1$ and $\xi=c \psi+d J \psi$. It follows that the family $t \mapsto \xi_{t}:=c \psi_{t}+d J \psi_{t}$ is defined for all $t \in[0,1]$. Thus, for every $L^{2}$ normalized eigenspinor $\xi \in \operatorname{ker}\left(D^{g}-\lambda\right)$ and for every $h \in V$ we can define $F_{\xi}(h)$. For every $i \in\{-m, \ldots, m\} \backslash\{0\}$ we obtain an open neighborhood $V \subset R(M)$ of $g$ as above and after taking the intersection of these open neighborhoods we may assume that $V$ is independent of $i$. The first assertion follows.

In order to show the second assertion let $h \in V$ and let $\left(k_{j}\right)_{j \in \mathbb{N}}$ be a sequence in $V$ such that $k_{j} \rightarrow h$ as $j \rightarrow \infty$. Let $i \in\{-m, \ldots, m\} \backslash\{0\}$, let $\psi$ be an eigenspinor of $D^{g}$ corresponding to $\lambda_{i}(g)$. We define the $L^{2}$-orthogonal projections

$$
\begin{aligned}
P_{j}: & C^{\infty}\left(\Sigma^{g} M\right) \rightarrow \operatorname{ker}\left(D^{g, k_{j}}-\lambda_{i}\left(k_{j}\right)\right), \quad j \in \mathbb{N} \\
P: & C^{\infty}\left(\Sigma^{g} M\right) \rightarrow \operatorname{ker}\left(D^{g, h}-\lambda_{i}(h)\right) .
\end{aligned}
$$

We regard the operators $D^{g, h}, D^{g, k_{j}}, j \in \mathbb{N}$, as closed operators on $C^{0}\left(\Sigma^{g} M\right)$ with domain $C^{1}\left(\Sigma^{g} M\right)$. Then by Theorem IV.3.16 in [20] we have $P_{j} \rightarrow P$ with respect to the norm of bounded linear operators on $C^{0}\left(\Sigma^{g} M\right)$. In particular for all $j \in \mathbb{N}$ large enough the spinor $\alpha_{j}:=\frac{P_{j}\left(F_{\psi}(h)\right)}{\left\|P_{j}\left(F_{\psi}(h)\right)\right\|_{L^{2}}}$ is well defined and we have $\left\|\alpha_{j}-F_{\psi}(h)\right\|_{C^{0}\left(\Sigma^{g} M\right)} \rightarrow 0$ as $j \rightarrow \infty$. By definition of $F_{\psi}$ we have for every $j \in \mathbb{N}$ :

$$
F_{\psi}\left(k_{j}\right)=\frac{\left(\psi, \alpha_{j}\right)_{L^{2}} \alpha_{j}-\left(\alpha_{j}, J \psi\right)_{L^{2}} J \alpha_{j}}{\left(\left|\left(\psi, \alpha_{j}\right)_{L^{2}}\right|^{2}+\left|\left(J \psi, \alpha_{j}\right)_{L^{2}}\right|^{2}\right)^{1 / 2}} .
$$

Let $x \in M$ and let $\left(x_{j}\right)_{j \in \mathbb{N}}$ be a sequence in $M$ such that $x_{j} \rightarrow x$ as $j \rightarrow \infty$. It follows that $F_{\psi}^{\mathrm{ev}}\left(k_{j}, x_{j}\right) \rightarrow F_{\psi}^{\mathrm{ev}}(h, x)$ as $j \rightarrow \infty$. The second assertion follows.

In order to prove the third assertion let $h, k \in V_{f_{1} \ldots f_{s}} \cap V$ and let $x \in M$. For $t \in[0,1]$ we define $h_{t}:=h+t(k-h)$ and $\psi_{t}:=F_{\psi}\left(h_{t}\right)$. The family $t \mapsto$ $\psi_{t}$ is real analytic. In order to see this we use that by Lemma 2.1 there exists a real analytic family $t \mapsto \alpha_{t} \in C^{\infty}\left(\Sigma^{h} M\right)$ of $L^{2}$-normalized eigenspinors of $D^{h, h_{t}}$. For all $t \in[0,1]$ we have $\bar{\beta}_{h, h_{t}}^{-1}=\bar{\beta}_{h_{t}, h}$ and thus

$$
\bar{\beta}_{h_{t}, g} \bar{\beta}_{h, h_{t}} D^{h, h_{t}}=D^{g, h_{t}} \bar{\beta}_{h_{t}, g} \bar{\beta}_{h, h_{t}} .
$$

Thus, the family $t \mapsto \bar{\beta}_{h_{t}, g} \bar{\beta}_{h, h_{t}} \alpha_{t} \in C^{\infty}\left(\Sigma^{g} M\right)$ is a real analytic family of $L^{2}$-normalized eigenspinors of $D^{g, h_{t}}$. The family $t \mapsto \psi_{t}$ is obtained from this 
family by a unitary transformation as above and therefore is real analytic. Thus, the derivative of $F_{\psi}^{\mathrm{ev}}$ at $(h, x)$ in the direction $k-h$ exists and is given by $\left.\frac{d \psi_{t}}{d t}\right|_{t=0}(x)$. In order to show continuity of the derivative let $\left(k_{j}\right)_{j \in \mathbb{N}}$ be a sequence in $V_{f_{1} \ldots f_{s}} \cap V$ such that $k_{j} \rightarrow h$ with respect to the $C^{1}$-topology as $j \rightarrow \infty$. After deleting finitely many of the $k_{j}$ we may assume that for every $j \in \mathbb{N}$ and for every $t \in[0,1]$ the tensor field $k_{j, t}:=k_{j}+t(k-h)$ is a Riemannian metric on $M$. Then we write $\psi_{t}^{k_{j}}:=F_{\psi}\left(k_{j, t}\right), h_{t}:=h+t(k-h)$ and $\psi_{t}^{h}:=F_{\psi}\left(h_{t}\right)$. For all $t \in[0,1]$ and for all $j \in \mathbb{N}$ we have

$$
\begin{gathered}
\operatorname{Re}\left(\psi_{t}^{k_{j}}, i \psi\right)_{L^{2}}=0, \quad\left(\psi_{t}^{k_{j}}, J \psi\right)_{L^{2}}=0, \quad\left(\psi_{t}^{k_{j}}, \psi_{t}^{k_{j}}\right)_{L^{2}}=1 \\
\operatorname{Re}\left(\psi_{t}^{h}, i \psi\right)_{L^{2}}=0, \quad\left(\psi_{t}^{h}, J \psi\right)_{L^{2}}=0, \quad\left(\psi_{t}^{h}, \psi_{t}^{h}\right)_{L^{2}}=1 .
\end{gathered}
$$

By taking the derivative at $t=0$ we conclude that for all $j \in \mathbb{N}$ we have

$$
\left.\frac{d \psi_{t}^{k_{j}}}{d t}\right|_{t=0} \in \operatorname{ker}\left(D^{g}-\lambda\right)^{\perp},\left.\quad \frac{d \psi_{t}^{h}}{d t}\right|_{t=0} \in \operatorname{ker}\left(D^{g}-\lambda\right)^{\perp} .
$$

For all $t \in[0,1]$ and for all $j \in \mathbb{N}$ we have

$$
\left(D^{g, k_{j, t}}-\lambda_{t}^{k_{j}}\right) \psi_{t}^{k_{j}}=0, \quad\left(D^{g, h_{t}}-\lambda_{t}^{h}\right) \psi_{t}^{h}=0 .
$$

By taking the derivative at $t=0$ we obtain for all $j \in \mathbb{N}$

$$
\left.\left(D^{g}-\lambda\right) \frac{d \psi_{t}^{k_{j}}}{d t}\right|_{t=0}=-\left(\left.\frac{d}{d t} D^{g, k_{j, t}}\right|_{t=0}-\left.\frac{d \lambda_{t}^{k_{j}}}{d t}\right|_{t=0}\right) \psi
$$

and analogously for $h$ instead of $k_{j}$. Since the formulas (2.2) and (2.3) for the derivatives of the Dirac operator and of the eigenvalue contain derivatives of the metric of order at most 1 and since $k_{j} \rightarrow h$ in the $C^{1}$-topology we obtain

$$
\left\|\left(\left.\frac{d}{d t} D^{g, h_{t}}\right|_{t=0}-\left.\frac{d \lambda_{t}^{h}}{d t}\right|_{t=0}\right) \psi-\left(\left.\frac{d}{d t} D^{g, k_{j, t}}\right|_{t=0}-\left.\frac{d \lambda_{t}^{k_{j}}}{d t}\right|_{t=0}\right) \psi\right\|_{C^{0}\left(\Sigma^{g} M\right)} \rightarrow 0
$$

as $j \rightarrow \infty$. By (4.3) we conclude

$$
\left\|\left(D^{g}-\lambda\right)\left(\left.\frac{d \psi_{t}^{k_{j}}}{d t}\right|_{t=0}-\left.\frac{d \psi_{t}^{h}}{d t}\right|_{t=0}\right)\right\|_{C^{0}\left(\Sigma^{g} M\right)} \rightarrow 0
$$


as $j \rightarrow \infty$. For $i=0,1$ we define

$$
U_{i}:=C^{i}\left(\Sigma^{g} M\right) \cap\left(\operatorname{ker}\left(D^{g}-\lambda\right)\right)^{\perp} .
$$

and equip this space with the $C^{i}$-norm. Then the operator

$$
\left.\left(D^{g}-\lambda\right)\right|_{U_{1}}: \quad U_{1} \rightarrow U_{0}
$$

is bounded and bijective. Thus its inverse is also bounded and from (4.2) and (4.4) we obtain

$$
\left\|\left.\frac{d \psi_{t}^{k_{j}}}{d t}\right|_{t=0}-\left.\frac{d \psi_{t}^{h}}{d t}\right|_{t=0}\right\|_{C^{1}\left(\Sigma^{g} M\right)} \rightarrow 0
$$

as $j \rightarrow \infty$. Let $\left(x_{j}\right)_{j \in \mathbb{N}}$ be a sequence in $M$ with $x_{j} \rightarrow x$ as $j \rightarrow \infty$. We obtain $\left.\left.\frac{d \psi_{t}^{k_{j}}}{d t}\right|_{t=0}\left(x_{j}\right) \rightarrow \frac{d \psi_{t}^{h}}{d t}\right|_{t=0}(x)$ and the third assertion follows.

Remark 4.5. Let $m \in \mathbb{N} \backslash\{0\}$ and let $g \in S_{m}(M)$, not necessarily $g \in$ $S_{0}(M, \Theta)$. There exists an open neighborhood $V \subset[g]$ of $g$ in $[g]$ with analogous properties as the neighborhood $V$ in Lemma 4.4. Namely for the proof of (4.1) we use that for all $h \in[g]$ we have $\operatorname{dim} \operatorname{ker}\left(D^{g}\right)=\operatorname{dim} \operatorname{ker}\left(D^{h}\right)$.

Lemma 4.6. Let $M$ be a closed spin manifold of dimension 2 or 3 and let $m \in \mathbb{N} \backslash\{0\}$. Then $S_{0}(M, \Theta) \cap N_{m}(M)$ is open in $R(M)$. Furthermore, for every $g \in R(M)$ the subset $N_{m}(M) \cap[g]$ is open in $[g]$.

Proof. Let $g \in S_{0}(M, \Theta) \cap N_{m}(M)$. Then we have $g \in S_{0}(M, \Theta) \cap S_{m}(M)$. We choose an open neighborhood $V \subset R(M)$ of $h$ as in Lemma 4.4. Then for every $i \in\{-m, \ldots, m\} \backslash\{0\}$ we choose an $L^{2}$-normalized eigenspinor $\psi_{i}$ of $D^{h}$ corresponding to $\lambda_{i}(h)$ and we define $F_{\psi_{i}}: V \rightarrow C^{\infty}\left(\Sigma^{h} M\right)$ as in Lemma 4.4. Since $F_{\psi_{i}}^{\mathrm{ev}}$ is continuous and the complement of the zero section is open in $\Sigma^{g} M$ it follows that for every $i$ the subset

$$
A_{\psi_{i}}:=\left\{h \in V \mid F_{\psi_{i}}(h) \text { is nowhere zero }\right\}
$$

is an open neighborhood of $g$ in $R(M)$. We observe that for every $i$ we have

$$
A_{\psi_{i}} \subset\left\{h \in V \mid \text { all eigenspinors to } \lambda_{i}(h) \text { are nowhere zero }\right\}
$$

since $\lambda_{i}(h)$ is a simple eigenvalue of $D^{g, h}$ and thus the zero sets of all eigenspinors corresponding to $\lambda_{i}(h)$ coincide. Then the intersection of the subsets 
$A_{\psi_{i}}, i \in\{-m, \ldots, m\} \backslash\{0\}$, is an open neighborhood of $g$ in $R(M)$ and is contained in $S_{0}(M, \Theta) \cap N_{m}(M)$. The first assertion follows. The proof of the second assertion is analogous.

The strategy for the proof of density in Theorem 4.3 is based on the following remark.

Remark 4.7. Let $A \subset \Sigma^{g} M$ be the zero section. The dimension of the total space $\Sigma^{g} M$ of the spinor bundle is

$$
\operatorname{dim} \Sigma^{g} M=n+2^{1+[n / 2]}>2 n=\operatorname{dim} M+\operatorname{dim} A,
$$

and thus a map $f: M \rightarrow \Sigma^{g} M$ is transverse to $A$ if and only if $f^{-1}(A)=\emptyset$.

Therefore in order to prove that $S_{0}(M, \Theta) \cap N_{m}(M)$ is dense in $R(M)$ and that for every $g \in R(M)$ the subset $N_{m}(M) \cap[g]$ is dense in $[g]$ we would like to apply Theorem 2.3. Our aim is then to show that a suitable restriction of the map $F_{\psi}^{\mathrm{ev}}$ defined as in Lemma 4.4 is transverse to the zero section.

Let $p \in M$ with $\psi(p)=0$. We have a canonical decomposition of the tangent space

$$
T_{\psi(p)} \Sigma^{g} M \cong \Sigma_{p}^{g} M \oplus T_{p} M
$$

and thus

$$
\left.d F_{\psi}^{\mathrm{ev}}\right|_{(g, p)}: \quad T_{g} V_{f_{1} \ldots f_{s}} \oplus T_{p} M \rightarrow \Sigma_{p}^{g} M \oplus T_{p} M
$$

For a given $h \in V_{f_{1} \ldots f_{s}}$ we will write $g_{t}=g+t(h-g)$ and

$$
\psi_{t}:=F_{\psi}\left(g_{t}\right),\left.\quad \frac{d \psi_{t}(x)}{d t}\right|_{t=0}:=\pi_{1}\left(\left.d F_{\psi}^{\mathrm{ev}}\right|_{(g, x)}(h-g, 0)\right)
$$

where $\pi_{1}$ is the projection onto the first summand. Then it follows that

$$
0=\left(\left.\frac{d}{d t} D^{g, g_{t}}\right|_{t=0}-\left.\frac{d \lambda_{t}}{d t}\right|_{t=0}\right) \psi+\left.\left(D^{g}-\lambda\right) \frac{d \psi_{t}}{d t}\right|_{t=0}
$$


Remark 4.8. Let $\varphi \in \Sigma_{p}^{g} M$ and $X, Y \in T_{p} M$. If one polarizes the identity

$$
\langle X \cdot \varphi, X \cdot \varphi\rangle=g(X, X)\langle\varphi, \varphi\rangle
$$

then one obtains

$$
\operatorname{Re}\langle X \cdot \varphi, Y \cdot \varphi\rangle=g(X, Y)\langle\varphi, \varphi\rangle
$$

Since Clifford multiplication with vectors is antisymmetric, it follows that $\operatorname{Re}\langle X \cdot \varphi, \varphi\rangle=0$. Let $\varphi \neq 0$ and let $\left(e_{i}\right)_{i=1}^{n}$ be an orthonormal basis of $T_{p} M$. It follows that for $n=2$ the spinors

$$
\varphi, e_{1} \cdot \varphi, e_{2} \cdot \varphi, e_{1} \cdot e_{2} \cdot \varphi
$$

form an orthogonal basis of $\Sigma_{p}^{g} M$ with respect to the real scalar product $\operatorname{Re}\langle.,$.$\rangle . Similarly for n=3$ the spinors

$$
\varphi, e_{1} \cdot \varphi, e_{2} \cdot \varphi, e_{3} \cdot \varphi
$$

form an orthogonal basis of $\Sigma_{p}^{g} M$ with respect to $\operatorname{Re}\langle.,$.$\rangle .$

The following rather long lemma is the most important step in showing that a suitable restriction of $F_{\psi}^{\text {ev }}$ is transverse to the zero section.

Lemma 4.9. Let $M$ be a closed connected spin manifold of dimension $n \in$ $\{2,3\}$ and let $m \in \mathbb{N} \backslash\{0\}$. Let $g \in S_{m}(M)$ and let $\lambda \in\left\{\lambda_{-m}(g), \ldots, \lambda_{m}(g)\right\}$. Let $\psi$ be an $L^{2}$-normalized eigenspinor of $D^{g}$ corresponding to $\lambda$ and let $p \in M$ with $\psi(p)=0$. Then there exist $f_{1}, \ldots, f_{4} \in C^{\infty}(M, \mathbb{R})$ such that the $\operatorname{map} F_{\psi}^{\mathrm{ev}}: V_{f_{1} \ldots f_{4}} \times M \rightarrow \Sigma^{g} M$ satisfies

$$
\pi_{1}\left(\left.d F_{\psi}^{\mathrm{ev}}\right|_{(g, p)}\left(T_{g} V_{f_{1} \ldots f_{4}} \oplus\{0\}\right)\right)=\Sigma_{p}^{g} M
$$

Proof. Assume that the claim is wrong. Then there exists $\varphi \in \Sigma_{p}^{g} M \backslash\{0\}$ such that for all $f \in C^{\infty}(M, \mathbb{R})$ we have

$$
0=\operatorname{Re}\left\langle\pi_{1}\left(\left.d F_{\psi}^{\mathrm{ev}}\right|_{(g, p)}(f g, 0)\right), \varphi\right\rangle=\operatorname{Re}\left\langle\left.\frac{d \psi_{t}}{d t}\right|_{t=0}(p), \varphi\right\rangle
$$


From the formula (3.7) for Green's function it follows that

$$
\begin{aligned}
0= & \operatorname{Re} \int_{M \backslash\{p\}}\left\langle\left.\left(D^{g}-\lambda\right) \frac{d \psi_{t}}{d t}\right|_{t=0}, G_{\lambda}^{g}(., p) \varphi\right\rangle \mathrm{dv}^{g} \\
& +\operatorname{Re}\left\langle P\left(\left.\frac{d \psi_{t}}{d t}\right|_{t=0}\right)(p), \varphi\right\rangle .
\end{aligned}
$$

Since $\lambda$ is a simple eigenvalue, all spinors in $\operatorname{ker}\left(D^{g}-\lambda\right)$ vanish at $p$. Thus the last term vanishes. By (3.6) and (4.5) we have

$$
\begin{aligned}
0 & =-\operatorname{Re} \int_{M \backslash\{p\}}\left\langle\left(\left.\frac{d}{d t} D^{g, g_{t}}\right|_{t=0}-\left.\frac{d \lambda_{t}}{d t}\right|_{t=0}\right) \psi, G_{\lambda}^{g}(., p) \varphi\right\rangle \mathrm{dv}^{g} \\
& =-\operatorname{Re} \int_{M \backslash\{p\}}\left\langle\left.\frac{d}{d t} D^{g, g_{t}}\right|_{t=0} \psi, G_{\lambda}^{g}(., p) \varphi\right\rangle \mathrm{dv}^{g}
\end{aligned}
$$

for all $f \in C^{\infty}(M, \mathbb{R})$. If we use the formula (2.2) for the derivative of the Dirac operator and

$$
\operatorname{grad}^{g}(f) \cdot \psi=\left(D^{g}-\lambda\right)(f \psi)
$$

it follows that

$$
\begin{aligned}
0= & \frac{1}{2} \operatorname{Re} \int_{M \backslash\{p\}} \lambda f\left\langle\psi, G_{\lambda}^{g}(., p) \varphi\right\rangle \mathrm{dv}^{g} \\
& +\frac{1}{4} \operatorname{Re} \int_{M \backslash\{p\}}\left\langle\left(D^{g}-\lambda\right)(f \psi), G_{\lambda}^{g}(., p) \varphi\right\rangle \mathrm{dv}^{g}
\end{aligned}
$$

Using the definition of Green's function and that all spinors in $\operatorname{ker}\left(D^{g}-\lambda\right)$ vanish at $p$, we find that

$$
\begin{aligned}
0 & =\frac{1}{2} \operatorname{Re} \int_{M \backslash\{p\}} \lambda f\left\langle\psi, G_{\lambda}^{g}(., p) \varphi\right\rangle \mathrm{dv}^{g}+\frac{1}{4} \operatorname{Re}\langle(f \psi)(p)-P(f \psi)(p), \varphi\rangle \\
& =\frac{1}{2} \operatorname{Re} \int_{M \backslash\{p\}} \lambda f\left\langle\psi, G_{\lambda}^{g}(., p) \varphi\right\rangle \mathrm{dv}^{g}
\end{aligned}
$$

for all $f \in C^{\infty}(M, \mathbb{R})$. Since we have $\lambda \neq 0$ it follows that $\operatorname{Re}\left\langle\psi, G_{\lambda}^{g}(., p) \varphi\right\rangle$ vanishes identically on $M \backslash\{p\}$.

Our aim is now to conclude that all the derivatives of $\psi$ at the point $p$ vanish. Then by Theorem 2.4 it follows that $\psi$ is identically zero, which is a contradiction. In order to show this we choose a local parametrization $\rho$ : $V \rightarrow U$ of $M$ by Riemannian normal coordinates, where $U \subset M$ is an open 
neighborhood of $p, V \subset \mathbb{R}^{n}$ is an open neighborhood of 0 and $\rho(0)=p$. Furthermore let

$$
\beta:\left.\left.\quad \Sigma \mathbb{R}^{n}\right|_{V} \rightarrow \Sigma^{g} M\right|_{U}, \quad A: \quad C^{\infty}\left(\left.\Sigma^{g} M\right|_{U}\right) \rightarrow C^{\infty}\left(\left.\Sigma \mathbb{R}^{n}\right|_{V}\right)
$$

denote the maps which send a spinor to its corresponding spinor in the Bourguignon-Gauduchon trivialization defined in Section 3.1. We show by induction that $\nabla^{r} A \psi(0)=0$ for all $r \in \mathbb{N}$, where $\nabla$ denotes the covariant derivative on $\Sigma \mathbb{R}^{n}$. The case $r=0$ is clear.

Let $r \geq 1$ and assume that we have $\nabla^{s} A \psi(0)=0$ for all $s \leq r-1$. Let $\left(E_{i}\right)_{i=1}^{n}$ be the standard basis of $\mathbb{R}^{n}$. First consider the case $n=2$. In the Bourguignon-Gauduchon trivialization we have

$$
A \psi(x)=\frac{1}{r !} \sum_{j_{1}, \ldots, j_{r}=1}^{2} x_{j_{1}} \ldots x_{j_{r}} \nabla_{E_{j_{1}}} \ldots \nabla_{E_{j_{r}}} A \psi(0)+O\left(|x|^{r+1}\right)
$$

by Taylor's formula and

$$
A\left(G_{\lambda}^{g}(., p) \varphi\right)(x)=-\frac{1}{2 \pi|x|^{2}} x \cdot \gamma-\frac{\lambda}{2 \pi} \ln |x| \gamma+O\left(|x|^{0}\right)
$$

by Theorem 3.11, where $\gamma:=\beta^{-1} \varphi \in \Sigma_{n}$ is the constant spinor on $\mathbb{R}^{n}$ corresponding to $\varphi$. It follows that

$$
\begin{aligned}
0= & -2 \pi r !|x|^{2} \operatorname{Re}\left\langle A\left(G_{\lambda}^{g}(., p) \varphi\right)(x), A \psi(x)\right\rangle \\
= & \sum_{i, j_{1}, \ldots, j_{r}=1}^{2} x_{i} x_{j_{1}} \ldots x_{j_{r}} \operatorname{Re}\left\langle E_{i} \cdot \gamma, \nabla_{E_{j_{1}}} \ldots \nabla_{E_{j_{r}}} A \psi(0)\right\rangle \\
& +\lambda \sum_{j_{1}, \ldots, j_{r}=1}^{2} x_{j_{1}} \ldots x_{j_{r}}|x|^{2} \ln |x| \operatorname{Re}\left\langle\gamma, \nabla_{E_{j_{1}}} \ldots \nabla_{E_{j_{r}}} A \psi(0)\right\rangle+O\left(|x|^{r+2}\right) .
\end{aligned}
$$

For all $k_{1}, k_{2} \in \mathbb{N}$ with $k_{1}+k_{2}=r+1$ the coefficient of $x_{1}^{k_{1}} x_{2}^{k_{2}}$ must be zero. This coefficient is obtained from the first sum on the right hand side and it is equal to

$$
\operatorname{Re}\left\langle E_{1} \cdot \gamma, \nabla_{E_{1}}^{k_{1}-1} \nabla_{E_{2}}^{k_{2}} A \psi(0)\right\rangle \frac{k_{1} r !}{k_{1} ! k_{2} !}+\operatorname{Re}\left\langle E_{2} \cdot \gamma, \nabla_{E_{1}}^{k_{1}} \nabla_{E_{2}}^{k_{2}-1} A \psi(0)\right\rangle \frac{k_{2} r !}{k_{1} ! k_{2} !}
$$


Thus if we write $x_{1}^{k_{1}} x_{2}^{k_{2}}=x_{i} x_{j_{1}} \ldots x_{j_{r}}$ with $i, j_{1}, \ldots, j_{r} \in\{1,2\}$, we obtain

$$
\begin{aligned}
0= & \frac{r !}{k_{1} ! k_{2} !}\left(\operatorname{Re}\left\langle E_{i} \cdot \gamma, \nabla_{E_{j_{1}}} \ldots \nabla_{E_{j_{r}}} A \psi(0)\right\rangle\right. \\
& \left.+\sum_{s=1}^{r} \operatorname{Re}\left\langle E_{j_{s}} \cdot \gamma, \nabla_{E_{i}} \nabla_{E_{j_{1}}} \ldots \widehat{\nabla_{E_{j_{s}}}} \ldots \nabla_{E_{j_{r}}} A \psi(0)\right\rangle\right)
\end{aligned}
$$

for all $i, j_{1}, \ldots, j_{r} \in\{1,2\}$, where the hat means that the operator is left out. For all $k_{1}, k_{2} \in \mathbb{N}$ with $k_{1}+k_{2}=r$ the coefficient of $x_{1}^{k_{1}+2} x_{2}^{k_{2}} \ln |x|$ in (4.7) must be zero. This coefficient is obtained from the second sum on the right hand side and it is equal to

$$
\frac{\lambda r !}{k_{1} ! k_{2} !} \operatorname{Re}\left\langle\gamma, \nabla_{E_{1}}^{k_{1}} \nabla_{E_{2}}^{k_{2}} A \psi(0)\right\rangle+\frac{\lambda r ! k_{2}\left(k_{2}-1\right)}{k_{1} ! k_{2} !} \operatorname{Re}\left\langle\gamma, \nabla_{E_{1}}^{k_{1}+2} \nabla_{E_{2}}^{k_{2}-2} A \psi(0)\right\rangle
$$

Using induction on $k_{2}$ and using the fact that $\lambda \neq 0$ we obtain for all $k_{1}, k_{2} \in$ $\mathbb{N}$ with $k_{1}+k_{2}=r$ that

$$
\operatorname{Re}\left\langle\gamma, \nabla_{E_{1}}^{k_{1}} \nabla_{E_{2}}^{k_{2}} A \psi(0)\right\rangle=0
$$

and therefore

$$
0=\operatorname{Re}\left\langle\gamma, \nabla_{E_{j_{1}}} \ldots \nabla_{E_{j_{r}}} A \psi(0)\right\rangle
$$

for all $j_{1}, \ldots, j_{r}, i \in\{1,2\}$. By (4.9) and Remark 4.8 there exist $a_{j_{1}, \ldots, j_{r}, k}$, $b_{j_{1}, \ldots, j_{r}} \in \mathbb{R}$ such that

$$
\nabla_{E_{j_{1}}} \ldots \nabla_{E_{j_{r}}} A \psi(0)=\sum_{k=1}^{2} a_{j_{1}, \ldots, j_{r}, k} E_{k} \cdot \gamma+b_{j_{1}, \ldots, j_{r}} E_{1} \cdot E_{2} \cdot \gamma
$$

Observe that the coefficients $a_{j_{1}, \ldots, j_{r}, k}$ are symmetric in the first $r$ indices. We insert this into (4.8) and we obtain

$$
0=a_{j_{1}, \ldots, j_{r}, i}+\sum_{k=1}^{r} a_{i, j_{1}, \ldots, \hat{j}_{k}, \ldots, j_{r}, j_{k}}
$$


for all $j_{1}, \ldots, j_{r}, i \in\{1,2\}$. On the other hand since $\psi \in \operatorname{ker}\left(D^{g}-\lambda\right)$ we find using the induction hypothesis

$$
\begin{aligned}
0= & \lambda \nabla_{E_{j_{1}}} \ldots \nabla_{E_{j_{r-1}}} A \psi(0) \\
= & \nabla_{E_{j_{1}}} \ldots \nabla_{E_{j_{r-1}}} \sum_{i=1}^{2} E_{i} \cdot \nabla_{E_{i}} A \psi(0) \\
= & \sum_{i, k=1}^{2} a_{j_{1}, \ldots, j_{r-1}, i, k} E_{i} \cdot E_{k} \cdot \gamma+\sum_{i=1}^{2} b_{j_{1}, \ldots, j_{r-1}, i} E_{i} \cdot E_{1} \cdot E_{2} \cdot \gamma \\
= & -\left(a_{j_{1}, \ldots, j_{r-1}, 1,1}+a_{j_{1}, \ldots, j_{r-1}, 2,2}\right) \gamma \\
& +\left(a_{j_{1}, \ldots, j_{r-1}, 1,2}-a_{j_{1}, \ldots, j_{r-1}, 2,1}\right) E_{1} \cdot E_{2} \cdot \gamma \\
& +b_{j_{1}, \ldots, j_{r-1}, 2} E_{1} \cdot \gamma-b_{j_{1}, \ldots, j_{r-1}, 1} E_{2} \cdot \gamma
\end{aligned}
$$

for all $j_{1}, \ldots, j_{r-1} \in\{1,2\}$. We conclude $b_{j_{1}, \ldots, j_{r}}=0$ for all $j_{1}, \ldots, j_{r} \in\{1,2\}$. Next consider $a_{j_{1}, \ldots, j_{r}, i}$ with fixed $j_{1}, \ldots, j_{r}, i \in\{1,2\}$. If we have $j_{k}=i$ for all $k \in\{1, \ldots, r\}$, then by (4.10) we know that $a_{j_{1}, \ldots, j_{r}, i}=0$. If there exists $k$ such that $j_{k} \neq i$ it follows from the coefficient of $E_{1} \cdot E_{2} \cdot \gamma$ in (4.11) that

$$
a_{i, j_{1}, \ldots \hat{j}_{k} \ldots, j_{r}, j_{k}}=a_{j_{1}, \ldots, j_{r}, i}
$$

Again (4.10) yields $a_{j_{1}, \ldots, j_{r}, i}=0$. We conclude that all $a_{j_{1}, \ldots, j_{r}, i}$ vanish and that $\nabla^{r} A \psi(0)=0$. This proves the assertion in the case $n=2$.

Next consider $n=3$. In the Bourguignon-Gauduchon trivialization we have

$$
\begin{aligned}
A \psi(x)= & \frac{1}{r !} \sum_{j_{1}, \ldots, j_{r}=1}^{3} x_{j_{1}} \ldots x_{j_{r}} \nabla_{E_{j_{1}}} \ldots \nabla_{E_{j_{r}}} A \psi(0) \\
& +\frac{1}{(r+1) !} \sum_{j_{1}, \ldots, j_{r}, i=1}^{3} x_{j_{1}} \ldots x_{j_{r}} x_{i} \nabla_{E_{j_{1}}} \ldots \nabla_{E_{j_{r}}} \nabla_{E_{i}} A \psi(0)+o\left(|x|^{r+1}\right)
\end{aligned}
$$

by Taylor's formula and

$$
A\left(G_{\lambda}^{g}(., p) \varphi\right)(x)=-\frac{1}{4 \pi|x|^{3}} x \cdot \gamma+\frac{\lambda}{4 \pi|x|} \gamma+o\left(|x|^{-s}\right)
$$


for every $s>0$ by Theorem 3.11, where $\gamma$ is as above. It follows that

$$
\begin{aligned}
0= & -4 \pi r !|x|^{3} \operatorname{Re}\left\langle A\left(G_{\lambda}^{g}(., p) \varphi\right)(x), A \psi(x)\right\rangle \\
= & \sum_{i, j_{1}, \ldots, j_{r}=1}^{3} x_{j_{1}} \ldots x_{j_{r}} x_{i} \operatorname{Re}\left\langle E_{i} \cdot \gamma, \nabla_{E_{j_{1}}} \ldots \nabla_{E_{j_{r}}} A \psi(0)\right\rangle \\
& +\frac{1}{r+1} \sum_{i, j_{1}, \ldots, j_{r}, m=1}^{3} x_{j_{1}} \ldots x_{j_{r}} x_{i} x_{m} \operatorname{Re}\left\langle E_{i} \cdot \gamma, \nabla_{E_{j_{1}}} \ldots \nabla_{E_{j_{r}}} \nabla_{E_{m}} A \psi(0)\right\rangle \\
& -\lambda \sum_{j_{1}, \ldots, j_{r}=1}^{3} x_{j_{1}} \ldots x_{j_{r}}|x|^{2} \operatorname{Re}\left\langle\gamma, \nabla_{E_{j_{1}}} \ldots \nabla_{E_{j_{r}}} A \psi(0)\right\rangle+o\left(|x|^{r+2}\right) .
\end{aligned}
$$

Analogously to the case $n=2$ we obtain from the first term on the right hand side

$$
\begin{aligned}
0= & \operatorname{Re}\left\langle E_{i} \cdot \gamma, \nabla_{E_{j_{1}}} \ldots \nabla_{E_{j_{r}}} A \psi(0)\right\rangle \\
& +\sum_{s=1}^{r} \operatorname{Re}\left\langle E_{j_{s}} \cdot \gamma, \nabla_{E_{i}} \nabla_{E_{j_{1}}} \ldots \widehat{\nabla_{E_{j_{s}}}} \ldots \nabla_{E_{j_{r}}} A \psi(0)\right\rangle .
\end{aligned}
$$

for all $j_{1}, \ldots, j_{r}, i \in\{1,2,3\}$, where the hat means that the operator is left out. Our next aim is to obtain an analogue of (4.9) from the second and third term on the right hand side of (4.12). It is more difficult than in the case $n=2$, since derivatives of both orders $r$ and $r+1$ appear. The equation (3.2) reads

$$
\begin{aligned}
\lambda A \psi= & D^{g_{\text {eucl }}} A \psi+\sum_{i, j=1}^{3}\left(B_{i}^{j}-\delta_{i}^{j}\right) E_{i} \cdot \nabla_{E_{j}} A \psi \\
& +\frac{1}{4} \sum_{i, j, k=1}^{3} \widetilde{\Gamma}_{i j}^{k} E_{i} \cdot E_{j} \cdot E_{k} \cdot A \psi .
\end{aligned}
$$

Using (3.3) and (3.4) and that $|A \psi(x)|_{g_{\text {eucl }}}=O\left(|x|^{r}\right)$ as $x \rightarrow 0$ we find

$$
\lambda A \psi=D^{g_{\text {eucl }}} A \psi+O\left(|x|^{r+1}\right)
$$

and therefore

$$
\nabla_{E_{j_{1}}} \ldots \nabla_{E_{j_{r}}} D^{g_{\text {eucl }}} A \psi(0)=\lambda \nabla_{E_{j_{1}}} \ldots \nabla_{E_{j_{r}}} A \psi(0)
$$


for all $j_{1}, \ldots, j_{r} \in\{1,2,3\}$. Using the equation (3.1) we find

$$
A \nabla_{e_{i}}^{g} \psi=\nabla_{E_{i}} A \psi+O\left(|x|^{r+1}\right), \quad A \nabla_{e_{i}}^{g} \nabla_{e_{j}}^{g} \psi=\nabla_{E_{i}} \nabla_{E_{j}} A \psi+O\left(|x|^{r}\right)
$$

for all $i, j \in\{1,2,3\}$. Since by definition $\left.d \rho\right|_{x} ^{-1}\left(e_{i}\right)=E_{i}+O\left(|x|^{2}\right)$ the second term in the local formula (2.4) for $\nabla^{*} \nabla$ vanishes at $p$ and therefore we find

$$
A \nabla^{*} \nabla \psi=-\sum_{i=1}^{3} \nabla_{E_{i}} \nabla_{E_{i}} A \psi+O\left(|x|^{r}\right) .
$$

From the Schrödinger-Lichnerowicz formula (2.5) it follows that

$$
\lambda^{2} A \psi-\frac{\text { scal }}{4} A \psi=-\sum_{i=1}^{3} \nabla_{E_{i}} \nabla_{E_{i}} A \psi+O\left(|x|^{r}\right)
$$

and thus

$$
\nabla_{E_{j_{1}}} \ldots \nabla_{E_{j_{r-1}}} \sum_{i=1}^{3} \nabla_{E_{i}} \nabla_{E_{i}} A \psi(0)=0
$$

for all $j_{1}, \ldots, j_{r-1} \in\{1,2,3\}$. Now recall the second and third term on the right hand side of (4.12)

$$
\begin{aligned}
0= & \frac{1}{r+1} \sum_{j_{1}, \ldots, j_{r}, i, m=1}^{3} x_{j_{1}} \ldots x_{j_{r}} x_{i} x_{m} \operatorname{Re}\left\langle E_{i} \cdot \gamma, \nabla_{E_{j_{1}}} \ldots \nabla_{E_{j_{r}}} \nabla_{E_{m}} A \psi(0)\right\rangle \\
& -\lambda \sum_{j_{1}, \ldots, j_{r}=1}^{3} x_{j_{1}} \ldots x_{j_{r}}|x|^{2} \operatorname{Re}\left\langle\gamma, \nabla_{E_{j_{1}}} \ldots \nabla_{E_{j_{r}}} A \psi(0)\right\rangle
\end{aligned}
$$

and let $k_{1}, k_{2}, k_{3} \in \mathbb{N}$ such that $k_{1}+k_{2}+k_{3}=r$. Then from the coefficient of $x_{1}^{k_{1}+2} x_{2}^{k_{2}} x_{3}^{k_{3}}$ we find

$$
\begin{aligned}
0= & \operatorname{Re}\left\langle E_{1} \cdot \gamma, \nabla_{E_{1}}^{k_{1}} \nabla_{E_{2}}^{k_{2}} \nabla_{E_{3}}^{k_{3}} \nabla_{E_{1}} A \psi(0)\right\rangle \frac{r !}{\left(k_{1}+1\right) ! k_{2} ! k_{3} !} \\
& +\operatorname{Re}\left\langle E_{2} \cdot \gamma, \nabla_{E_{1}}^{k_{1}} \nabla_{E_{2}}^{k_{2}-1} \nabla_{E_{3}}^{k_{3}} \nabla_{E_{1}}^{2} A \psi(0)\right\rangle \frac{r ! k_{2}}{\left(k_{1}+2\right) ! k_{2} ! k_{3} !}
\end{aligned}
$$




$$
\begin{aligned}
& +\operatorname{Re}\left\langle E_{3} \cdot \gamma, \nabla_{E_{1}}^{k_{1}} \nabla_{E_{2}}^{k_{2}} \nabla_{E_{3}}^{k_{3}-1} \nabla_{E_{1}}^{2} A \psi(0)\right\rangle \frac{r ! k_{3}}{\left(k_{1}+2\right) ! k_{2} ! k_{3} !} \\
& -\lambda \operatorname{Re}\left\langle\gamma, \nabla_{E_{1}}^{k_{1}} \nabla_{E_{2}}^{k_{2}} \nabla_{E_{3}}^{k_{3}} A \psi(0)\right\rangle \frac{r !}{k_{1} ! k_{2} ! k_{3} !} \\
& -\lambda \operatorname{Re}\left\langle\gamma, \nabla_{E_{1}}^{k_{1}} \nabla_{E_{2}}^{k_{2}-2} \nabla_{E_{3}}^{k_{3}} \nabla_{E_{1}}^{2} A \psi(0)\right\rangle \frac{r ! k_{2}\left(k_{2}-1\right)}{\left(k_{1}+2\right) ! k_{2} ! k_{3} !} \\
& -\lambda \operatorname{Re}\left\langle\gamma, \nabla_{E_{1}}^{k_{1}} \nabla_{E_{2}}^{k_{2}} \nabla_{E_{3}}^{k_{3}-2} \nabla_{E_{1}}^{2} A \psi(0)\right\rangle \frac{r ! k_{3}\left(k_{3}-1\right)}{\left(k_{1}+2\right) ! k_{2} ! k_{3} !}
\end{aligned}
$$

From the coefficient of $x_{1}^{k_{1}} x_{2}^{k_{2}+2} x_{3}^{k_{3}}$ we find

$$
\begin{aligned}
0= & \operatorname{Re}\left\langle E_{1} \cdot \gamma, \nabla_{E_{1}}^{k_{1}-1} \nabla_{E_{2}}^{k_{2}} \nabla_{E_{3}}^{k_{3}} \nabla_{E_{2}}^{2} A \psi(0)\right\rangle \frac{r ! k_{1}}{k_{1} !\left(k_{2}+2\right) ! k_{3} !} \\
& +\operatorname{Re}\left\langle E_{2} \cdot \gamma, \nabla_{E_{1}}^{k_{1}} \nabla_{E_{2}}^{k_{2}} \nabla_{E_{3}}^{k_{3}} \nabla_{E_{2}} A \psi(0)\right\rangle \frac{r !}{k_{1} !\left(k_{2}+1\right) ! k_{3} !} \\
& +\operatorname{Re}\left\langle E_{3} \cdot \gamma, \nabla_{E_{1}}^{k_{1}} \nabla_{E_{2}}^{k_{2}} \nabla_{E_{3}}^{k_{3}-1} \nabla_{E_{2}}^{2} A \psi(0)\right\rangle \frac{r ! k_{3}}{k_{1} !\left(k_{2}+2\right) ! k_{3} !} \\
& -\lambda \operatorname{Re}\left\langle\gamma, \nabla_{E_{1}}^{k_{1}-2} \nabla_{E_{2}}^{k_{2}} \nabla_{E_{3}}^{k_{3}} \nabla_{E_{2}}^{2} A \psi(0)\right\rangle \frac{r ! k_{1}\left(k_{1}-1\right)}{k_{1} !\left(k_{2}+2\right) ! k_{3} !} \\
& -\lambda \operatorname{Re}\left\langle\gamma, \nabla_{E_{1}}^{k_{1}} \nabla_{E_{2}}^{k_{2}} \nabla_{E_{3}}^{k_{3}} A \psi(0)\right\rangle \frac{r !}{k_{1} ! k_{2} ! k_{3} !} \\
& -\lambda \operatorname{Re}\left\langle\gamma, \nabla_{E_{1}}^{k_{1}} \nabla_{E_{2}}^{k_{2}} \nabla_{E_{3}}^{k_{3}-2} \nabla_{E_{2}}^{2} A \psi(0)\right\rangle \frac{r ! k_{3}\left(k_{3}-1\right)}{k_{1} !\left(k_{2}+2\right) ! k_{3} !}
\end{aligned}
$$

From the coefficient of $x_{1}^{k_{1}} x_{2}^{k_{2}} x_{3}^{k_{3}+2}$ we find

$$
\begin{aligned}
0= & \operatorname{Re}\left\langle E_{1} \cdot \gamma, \nabla_{E_{1}}^{k_{1}-1} \nabla_{E_{2}}^{k_{2}} \nabla_{E_{3}}^{k_{3}} \nabla_{E_{3}}^{2} A \psi(0)\right\rangle \frac{r ! k_{1}}{k_{1} ! k_{2} !\left(k_{3}+2\right) !} \\
& +\operatorname{Re}\left\langle E_{2} \cdot \gamma, \nabla_{E_{1}}^{k_{1}} \nabla_{E_{2}}^{k_{2}-1} \nabla_{E_{3}}^{k_{3}} \nabla_{E_{3}}^{2} A \psi(0)\right\rangle \frac{r k_{2}}{k_{1} ! k_{2} !\left(k_{3}+2\right) !} \\
& +\operatorname{Re}\left\langle E_{3} \cdot \gamma, \nabla_{E_{1}}^{k_{1}} \nabla_{E_{2}}^{k_{2}} \nabla_{E_{3}}^{k_{3}} \nabla_{E_{3}} A \psi(0)\right\rangle \frac{r !}{k_{1} ! k_{2} !\left(k_{3}+1\right) !} \\
& -\lambda \operatorname{Re}\left\langle\gamma, \nabla_{E_{1}}^{k_{1}-2} \nabla_{E_{2}}^{k_{2}} \nabla_{E_{3}}^{k_{3}} \nabla_{E_{3}}^{2} A \psi(0)\right\rangle \frac{r ! k_{1}\left(k_{1}-1\right)}{k_{1} ! k_{2} !\left(k_{3}+2\right) !} \\
& -\lambda \operatorname{Re}\left\langle\gamma, \nabla_{E_{1}}^{k_{1}} \nabla_{E_{2}}^{k_{2}-2} \nabla_{E_{3}}^{k_{3}} \nabla_{E_{3}}^{2} A \psi(0)\right\rangle \frac{r ! k_{2}\left(k_{2}-1\right)}{k_{1} ! k_{2} !\left(k_{3}+2\right) !} \\
& -\lambda \operatorname{Re}\left\langle\gamma, \nabla_{E_{1}}^{k_{1}} \nabla_{E_{2}}^{k_{2}} \nabla_{E_{3}}^{k_{3}} A \psi(0)\right\rangle \frac{r !}{k_{1} ! k_{2} ! k_{3} !}
\end{aligned}
$$


We multiply the first equation with $\frac{\left(k_{1}+2\right) ! k_{2} ! k_{3} !}{r^{\prime} !}$, the second equation with $\frac{k_{1} !\left(k_{2}+2\right) ! k_{3} !}{r !}$ and the third equation with $\frac{k_{1} ! k_{2} !\left(k_{3}+2\right) !}{r !}$ and then add the multiplied equations. If we consider the lines with the same Roman numbers separately and use (4.14) and (4.15), then we find

$$
\begin{aligned}
0= & -2 \lambda \operatorname{Re}\left\langle\gamma, \nabla_{E_{1}}^{k_{1}} \nabla_{E_{2}}^{k_{2}} \nabla_{E_{3}}^{k_{3}} A \psi(0)\right\rangle \\
& +\operatorname{Re}\left\langle E_{1} \cdot \gamma, \nabla_{E_{1}}^{k_{1}} \nabla_{E_{2}}^{k_{2}} \nabla_{E_{3}}^{k_{3}} \nabla_{E_{1}} A \psi(0)\right\rangle k_{1} \\
& +\operatorname{Re}\left\langle E_{2} \cdot \gamma, \nabla_{E_{1}}^{k_{1}} \nabla_{E_{2}}^{k_{2}} \nabla_{E_{3}}^{k_{3}} \nabla_{E_{2}} A \psi(0)\right\rangle k_{2} \\
& +\operatorname{Re}\left\langle E_{3} \cdot \gamma, \nabla_{E_{1}}^{k_{1}} \nabla_{E_{2}}^{k_{2}} \nabla_{E_{3}}^{k_{3}} \nabla_{E_{3}} A \psi(0)\right\rangle k_{3} \\
& -\operatorname{Re}\left\langle E_{1} \cdot \gamma, \nabla_{E_{1}}^{k_{1}} \nabla_{E_{2}}^{k_{2}} \nabla_{E_{3}}^{k_{3}} \nabla_{E_{1}} A \psi(0)\right\rangle k_{1} \\
& -\operatorname{Re}\left\langle E_{2} \cdot \gamma, \nabla_{E_{1}}^{k_{1}} \nabla_{E_{2}}^{k_{2}} \nabla_{E_{3}}^{k_{3}} \nabla_{E_{2}} A \psi(0)\right\rangle k_{2} \\
& -\operatorname{Re}\left\langle E_{3} \cdot \gamma, \nabla_{E_{1}}^{k_{1}} \nabla_{E_{2}}^{k_{2}} \nabla_{E_{3}}^{k_{3}} \nabla_{E_{3}} A \psi(0)\right\rangle k_{3} \\
& -\lambda \operatorname{Re}\left\langle\gamma, \nabla_{E_{1}}^{k_{1}} \nabla_{E_{2}}^{k_{2}} \nabla_{E_{3}}^{k_{3}} A \psi(0)\right\rangle \sum_{i=1}^{3}\left(k_{i}+2\right)\left(k_{i}+1\right) \\
& +\lambda \operatorname{Re}\left\langle\gamma, \nabla_{E_{1}}^{k_{1}} \nabla_{E_{2}}^{k_{2}} \nabla_{E_{3}}^{k_{3}} A \psi(0)\right\rangle k_{1}\left(k_{1}-1\right) \\
& +\lambda \operatorname{Re}\left\langle\gamma, \nabla_{E_{1}}^{k_{1}} \nabla_{E_{2}}^{k_{2}} \nabla_{E_{3}}^{k_{3}} A \psi(0)\right\rangle k_{2}\left(k_{2}-1\right) \\
& +\lambda \operatorname{Re}\left\langle\gamma, \nabla_{E_{1}}^{k_{1}} \nabla_{E_{2}}^{k_{2}} \nabla_{E_{3}}^{k_{3}} A \psi(0)\right\rangle k_{3}\left(k_{3}-1\right)
\end{aligned}
$$

Therefore we obtain the analogue of (4.9) namely

$$
\operatorname{Re}\left\langle\gamma, \nabla_{E_{j_{1}}} \ldots \nabla_{E_{j_{r}}} A \psi(0)\right\rangle=0
$$

for all $j_{1}, \ldots, j_{r} \in\{1,2,3\}$. Thus there exist $a_{j_{1}, \ldots, j_{r}, k} \in \mathbb{R}$ such that

$$
\nabla_{E_{j_{1}}} \ldots \nabla_{E_{j_{r}}} A \psi(0)=\sum_{k=1}^{3} a_{j_{1}, \ldots, j_{r}, k} E_{k} \cdot \gamma
$$

Observe that the coefficients $a_{j_{1}, \ldots, j_{r}, k}$ are symmetric in the first $r$ indices. We insert this into (4.13) and we obtain

$$
0=a_{j_{1}, \ldots, j_{r}, i}+\sum_{k=1}^{r} a_{i, j_{1}, \ldots, \hat{j}_{k}, \ldots, j_{r}, j_{k}}
$$


for all $j_{1}, \ldots, j_{r}, i \in\{1,2,3\}$. On the other hand since $\psi \in \operatorname{ker}\left(D^{g}-\lambda\right)$ we find using the induction hypothesis

$$
\begin{aligned}
0 & =\lambda \nabla_{E_{j_{1}}} \ldots \nabla_{E_{j_{r-1}}} A \psi(0) \\
& =\nabla_{E_{j_{1}}} \ldots \nabla_{E_{j_{r-1}}} \sum_{i=1}^{3} E_{i} \cdot \nabla_{E_{i}} A \psi(0) \\
& =\sum_{i, k=1}^{3} a_{j_{1}, \ldots, j_{r-1}, i, k} E_{i} \cdot E_{k} \cdot \gamma \\
& =-\sum_{i=1}^{3} a_{j_{1}, \ldots, j_{r-1}, i, i} \gamma+\sum_{\substack{i, k=1 \\
i<k}}^{3}\left(a_{j_{1}, \ldots, j_{r-1}, i, k}-a_{j_{1}, \ldots, j_{r-1}, k, i}\right) E_{i} \cdot E_{k} \cdot \gamma
\end{aligned}
$$

for all $j_{1}, \ldots, j_{r-1} \in\{1,2,3\}$. Consider $a_{j_{1}, \ldots, j_{r}, i}$ with $j_{1}, \ldots, j_{r}, i \in\{1,2,3\}$. If $j_{k}=i$ for all $k \in\{1, \ldots, r\}$ then by (4.16) we know that $a_{j_{1}, \ldots, j_{r}, i}=0$. If there exists $k$ such that $j_{k} \neq i$ it follows from the coefficient of $E_{j_{k}} \cdot E_{i} \cdot \gamma$ in (4.17) that

$$
a_{i, j_{1}, \ldots, \hat{j}_{k} \ldots, j_{r}, j_{k}}=a_{j_{1}, \ldots, j_{r}, i}
$$

Again (4.16) yields $a_{j_{1}, \ldots, j_{r}, i}=0$. We conclude that all $a_{j_{1}, \ldots, j_{r}, i}$ vanish and that $\nabla^{r} A \psi(0)=0$. This proves the assertion in the case $n=3$.

Remark 4.10. It is not clear how to prove this lemma for $n \geq 4$. Namely the condition $\operatorname{Re}\left\langle\gamma, \nabla_{E_{i}} A \psi(0)\right\rangle=0$ for all $i$ leads to

$$
\nabla_{E_{i}} A \psi(0)=\sum_{k=1}^{n} a_{i k} E_{k} \cdot \gamma+\sum_{k=1}^{n} b_{i k} \cdot \gamma
$$

with $a_{i k} \in \mathbb{R}$ and with elements $b_{i k}$ of the Clifford algebra. As in (4.8), (4.13) with $r=1$ it follows that $a_{i k}=-a_{k i}$ for all $i, k$ and furthermore

$$
\begin{aligned}
0 & =\lambda A \psi(0) \\
& =\sum_{i=1}^{n} E_{i} \cdot \nabla_{E_{i}} A \psi(0) \\
& =2 \sum_{\substack{i, k=1 \\
i<k}}^{n} a_{i k} E_{i} \cdot E_{k} \cdot \gamma+\sum_{i, k=1}^{n} E_{i} \cdot b_{i k} \cdot \gamma
\end{aligned}
$$


But for $n \geq 4$ the spinors $E_{1} \cdot E_{2} \cdot \gamma$ and $E_{3} \cdot E_{4} \cdot \gamma$ are not linearly independent in general. Thus we cannot conclude immediately that all the $a_{i k}$ vanish.

Proof of Theorem 4.3. First we prove that $S_{0}(M, \Theta) \cap N_{m}(M)$ is dense in $R(M)$. Let $U \subset R(M)$ be open. It is sufficient to show that $U \cap S_{0}(M, \Theta) \cap$ $N_{m}(M)$ is not empty. Since by Dahl's result $S_{0}(M, \Theta) \cap S_{m}(M)$ is dense in $R(M)$, there exists a metric $g$ in $U \cap S_{0}(M, \Theta) \cap S_{m}(M)$. Let $V \subset R(M)$ be an open neighborhood of $g$ as in Lemma 4.4. We may assume that $V \subset$ $U \cap S_{0}(M, \Theta)$. Let $\lambda$ be one of the eigenvalues $\left\{\lambda_{-m}(g), \ldots, \lambda_{m}(g)\right\}$ of $D^{g}$ and let $\psi$ be an $L^{2}$-normalized eigenspinor corresponding to $\lambda$. Define

$$
F_{\psi}: \quad V \rightarrow C^{\infty}\left(\Sigma^{g} M\right)
$$

as in Lemma 4.4. We show that a suitable restriction of $F_{\psi}^{\text {ev }}$ is transverse to the zero section of $\Sigma^{g} M$. Let $p \in M$ with $\psi(p)=0$. By Lemma 4.9 there exist $f_{p, 1}, \ldots, f_{p, 4} \in C^{\infty}(M, \mathbb{R})$ such that we have

$$
\pi_{1}\left(\left.d F_{\psi}^{\mathrm{ev}}\right|_{(g, p)}\left(T_{g} V_{f_{p, 1}, \ldots, f_{p, 4}} \oplus\{0\}\right)\right)=\Sigma_{p}^{g} M
$$

By continuity of $d F_{\psi}^{\mathrm{ev}}$ there exists an open neighborhood $U_{p} \subset M$ of $p$ such that for all $q \in U_{p}$ with $\psi(q)=0$ we have

$$
\pi_{1}\left(\left.d F_{\psi}^{\mathrm{ev}}\right|_{(g, q)}\left(T_{g} V_{f_{p, 1}, \ldots, f_{p, 4}} \oplus\{0\}\right)\right)=\Sigma_{q}^{g} M
$$

For every $p \in M$ with $\psi(p)=0$ we choose an open neighborhood $U_{p} \subset M$ as above. Since the zero set of $\psi$ is compact, there exist finitely many points $p_{1}, \ldots, p_{r} \in M$ and open neighborhoods $U_{p_{i}} \subset M$ of $p_{i}$ and $f_{p_{i}, 1}, \ldots, f_{p_{i}, 4} \in$ $C^{\infty}(M, \mathbb{R}), 1 \leq i \leq r$, such that for every $i$ we have $\psi\left(p_{i}\right)=0$ and such that the open neighborhoods $U_{p_{1}}, \ldots, U_{p_{r}}$ cover the zero set of $\psi$ and such that for every $i \in\{1, \ldots, r\}$ and for every $q \in U_{p_{i}}$ with $\psi(q)=0$ we have

$$
\pi_{1}\left(\left.d F_{\psi}^{\mathrm{ev}}\right|_{(g, q)}\left(T_{g} V_{f_{p_{i}, 1}, \ldots, f_{p_{i}, 4}} \oplus\{0\}\right)\right)=\Sigma_{q}^{g} M
$$

We label the functions $f_{p_{i}, j}$ by $f_{1}, \ldots, f_{4 r}$. For every one of the finitely many eigenvalues $\left\{\lambda_{-m}(g), \ldots, \lambda_{m}(g)\right\}$ of $D^{g}$ we choose an eigenspinor and we repeat this procedure. Then for every $i \in\{-m, \ldots, m\} \backslash\{0\}$ we obtain functions $f_{i, 1}, \ldots, f_{i, 4 r_{i}}$ as above, where $r_{i} \in \mathbb{N}$ might depend on $i$. We label 
these functions by

$$
\left\{f_{1}, \ldots, f_{s}\right\}:=\bigcup_{\substack{i=-m \\ i \neq 0}}^{m}\left\{f_{i, 1}, \ldots, f_{i, 4 r_{i}}\right\} .
$$

We define

$$
V_{f_{1}, \ldots, f_{s}}:=\left\{\left(1+\sum_{i=1}^{s} t_{i} f_{i}\right) g \mid t_{1}, \ldots, t_{s} \in \mathbb{R}\right\} \cap V .
$$

and we define the maps $F_{\psi_{i}}: V_{f_{1}, \ldots, f_{s}} \rightarrow C^{\infty}\left(\Sigma^{g} M\right), i \in\{-m, \ldots, m\} \backslash\{0\}$, as in Lemma 4.4. Then for every $i \in\{-m, \ldots, m\} \backslash\{0\}$ and for every $q \in M$ with $\psi_{i}(q)=0$ we have

$$
\pi_{1}\left(\left.d F_{\psi_{i}}^{\mathrm{ev}}\right|_{(g, q)}\left(T_{g} V_{f_{1}, \ldots, f_{s}} \oplus\{0\}\right)\right)=\Sigma_{q}^{g} M .
$$

By continuity of the maps $d F_{\psi_{i}}^{\mathrm{ev}}$ there exists an open neighborhood $W \subset$ $V_{f_{1}, \ldots, f_{s}}$ of $g$ such that for every $i \in\{-m, \ldots, m\} \backslash\{0\}$ and for every $(h, q) \in$ $W \times M$ with $F_{\psi_{i}}^{\mathrm{ev}}(h, q)=0$ we have

$$
\pi_{1}\left(\left.d F_{\psi_{i}}^{\mathrm{ev}}\right|_{(h, q)}\left(T_{h} V_{f_{1}, \ldots, f_{s}} \oplus\{0\}\right)\right)=\Sigma_{q}^{g} M .
$$

It follows that the restrictions of all the maps $F_{\psi_{i}}^{\text {ev }}$ to $W \times M$ are transverse to the zero section of $\Sigma^{g} M$.

For every $i \in\{-m, \ldots, m\} \backslash\{0\}$ we define $Y_{i}$ as the subset of all $h \in W$ such that $F_{\psi_{i}}(h)$ is nowhere zero on $M$. By Remark 4.7 the subset $Y_{i}$ is the subset of all $h \in W$ such that $F_{\psi_{i}}(h)$ is transverse to the zero section. By Lemma 4.4 the restrictions of the maps $F_{\psi_{i}}^{\mathrm{ev}}$ to $W \times M$ are in $C^{1}(W \times$ $\left.M, \Sigma^{g} M\right)$ and thus the condition $r>0$ in Theorem 2.3 is satisfied. By this theorem all the subsets $Y_{i}$ are dense in $W$. Since the zero section is closed in $\Sigma^{g} M$ and all the maps $F_{\psi_{i}}^{\text {ev }}$ are continuous, all the subsets $Y_{i}$ are open in $W$. Thus the intersection

$$
Y:=\bigcap_{\substack{i=-m \\ i \neq 0}}^{m} Y_{i}
$$

is open and dense in $W$. By definition we have $Y=N_{m}(M) \cap W$. Since we have $W \subset U$ the intersection $S_{0}(M, \Theta) \cap N_{m}(M) \cap U$ is not empty. Thus $S_{0}(M, \Theta) \cap N_{m}(M)$ is dense in $R(M)$. The proof that for every $g$ in $R(M)$ the subset $N_{m}(M) \cap[g]$ is dense in $[g]$ is analogous if we use that $S_{m}(M) \cap[g]$ is dense in $[g]$ by Dahl's result. 


\subsection{Examples on closed surfaces}

In this section we give a counterexample showing that Theorem 1.1 does not hold for harmonic spinors in the case $n=2$. Let $(M, g, \Theta)$ be a closed Riemannian spin manifold of dimension 2. The spinor bundle splits as

$$
\Sigma^{g} M=\Sigma^{+} M \oplus \Sigma^{-} M
$$

and sections of $\Sigma^{ \pm} M$ will be called positive respectively negative spinors. The manifold $(M, g)$ is Kähler and the bundle $\Sigma^{+} M$ is canonically isomorphic to a holomorphic line bundle $L$ on $M$. Furthermore positive harmonic spinors can be identified with holomorphic sections of $L$ (see e.g., [8, 19]).

To every positive or negative spinor on $(M, g)$ one can assign a tangent vector field on $M$ by a method given in [1] which we briefly recall. First we define $\tau_{ \pm}: \mathrm{SO}(2) \rightarrow \mathbb{C}$ by

$$
\left(\begin{array}{cc}
\cos t & -\sin t \\
\sin t & \cos t
\end{array}\right) \mapsto \exp ( \pm \mathrm{i} t) .
$$

We define a complex structure $J$ on $M$ such that for every $p \in M$ and for every unit vector $X \in T_{p} M$ the system $(X, J X)$ is a positively oriented orthonormal basis of $T_{p} M$. Then the map $\mathrm{P}_{\mathrm{SO}}(M, g) \times_{\tau_{+}} \mathbb{C} \rightarrow(T M, J)$ which sends $\left[\left(e_{1}, e_{2}\right), 1\right]$ to $e_{1}$ is an isomorphism of complex line bundles. In the same way we obtain an isomorphism $\mathrm{P}_{\mathrm{SO}}(M, g) \times_{\tau_{-}} \mathbb{C} \rightarrow(T M,-J)$. Then the following holds.

Lemma $4.11[\mathbf{1}]$. Let $(M, g, \Theta)$ be a Riemannian spin manifold of dimension 2. Then the map

$$
\begin{gathered}
\Phi_{ \pm}: \quad \Sigma^{ \pm} M=\mathrm{P}_{\mathrm{Spin}}(M, g) \times_{\rho} \Sigma_{2}^{ \pm} \rightarrow \mathrm{P}_{\mathrm{SO}}(M, g) \times_{\tau_{\mp}} \mathbb{C} \cong(T M, \mp J) \\
{[s, \sigma] \mapsto\left[\Theta(s), \sigma^{2}\right]}
\end{gathered}
$$

is well defined.

We denote by $\gamma$ the genus of $M$. Assume that $\psi$ is a non-trivial positive harmonic spinor on $M$ and that $p \in M$ is a point with $\psi(p)=0$. After a choice of a local holomorphic chart of $M$ and of a local trivialization of the holomorphic line bundle $\Sigma^{+} M$ around $p$ the spinor $\psi$ corresponds locally to a holomorphic function. We define $m_{p}$ as the order of the zero $p$. Let $X$ be the vector field on $M$ associated with $\psi$ via Lemma 4.11. It follows that $X$ has an isolated zero at $p$ with index equal to $-2 m_{p}$. Let $\chi(M)=2-2 \gamma$ 
denote the Euler characteristic of $M$. Denote by $N$ the zero set of $\psi$. Since $M$ is compact, the set $N$ is finite. By the Poincaré-Hopf Theorem we obtain the following result.

Theorem 4.12. Assume that $\psi$ is a positive harmonic spinor on a closed surface $(M, g, \Theta)$ and let $N \subset M$ be its zero set. Then $N$ is finite and we have

$$
\sum_{p \in N} m_{p}=-\frac{1}{2} \chi(M)=\gamma-1
$$

On every closed oriented surface $M$ of genus 2 there exists a spin structure, such that for every metric $g$ on $M$ we have $\operatorname{dim}_{\mathbb{C}} \operatorname{ker}\left(D^{g}\right)=2$ (see Proposition 2.3 in [19] and its proof). We take such a spin structure. Then by Theorem 4.12 for every Riemannian metric $g$ on $M$ every positive harmonic spinor of $D^{g}$ vanishes at exactly one point. Thus Theorem 1.1 does not hold for harmonic spinors in the case $n=2$.

\section{Acknowledgments}

The author thanks Bernd Ammann and Mattias Dahl for many interesting discussions.

\section{References}

[1] B. Ammann, Spin-Strukturen und das Spektrum des Dirac-Operators, Dissertation zur Erlangung des Doktorgrades, Freiburg im Breisgau, 1998.

[2] B. Ammann, The smallest Dirac eigenvalue in a spin-conformal class and cmc-immersions, Comm. Anal. Geom. 17 (2009), 429-479.

[3] B. Ammann, A. Moroianu and S. Moroianu, The Cauchy problems for Einstein metrics and parallel spinors, Commun. Math. Phys. 320 (2013), 173-198.

[4] B. Ammann, M. Dahl and E. Humbert, Harmonic spinors and local deformations of the metric, Math. Res. Lett. 18 (2011), 927-936.

[5] B. Ammann and E. Humbert, Positive mass theorem for the Yamabe problem on spin manifolds, GAFA 15 (2005), 567-576. 
[6] N. Aronszajn, A unique continuation theorem for solutions of elliptic partial differential equations or inequalities of second order, J. Math. Pures Appl. 36 (1957), 235-249.

[7] M. Abramowitz and I. Stegun (eds.), Handbook of mathematical functions, Dover Publications, New York, 1964.

[8] C. Bär and P. Schmutz, Harmonic spinors on Riemann surfaces, Ann. Global Anal. Geom. 10 (1992), 263-273.

[9] C. Bär, The Dirac operator on space forms of positive curvature, J. Math. Soc. Japan 48 (1996), 69-83.

[10] C. Bär, Metrics with harmonic spinors, Geom. Func. Anal. 6 (1996), 899-942.

[11] C. Bär, On nodal sets for Dirac and Laplace operators, Comm. Math. Phys. 188(3) (1997), 709-721.

[12] J.-P. Bourguignon and P. Gauduchon, Spineurs, opérateurs de Dirac et variations de métriques, Comm. Math. Phys. 144(3) (1992), 581-599.

[13] M. Dahl, Dirac eigenvalues for generic metrics on three-manifolds, Ann. Global Anal. Geom. 24(1) (2003), 95-100.

[14] T. Friedrich, Zur Abhängigkeit des Dirac-Operators von der SpinStruktur, Colloq. Math. 48(1) (1984), 57-62.

[15] T. Friedrich, Dirac Operators in Riemannian geometry, American Mathematical Society, Providence, 2000.

[16] J. Frauendiener, J. M. Nester and L.B. Szabados, Witten spinors on maximal, conformally flat hypersurfaces, Class. Quantum Grav. 28 (2011), 185004.

[17] O. Hijazi, A conformal lower bound for the smallest eigenvalue of the Dirac operator and Killing spinors, Comm. Math. Phys. 104(1) (1986), 151-162.

[18] M. Hirsch, Differential topology, Springer Verlag, New York, 1976.

[19] N. Hitchin, Harmonic spinors, Adv. Math. 14 (1974), 1-55.

[20] T. Kato, Perturbation theory of linear operators, Reprint of the 1980 edn., Springer Verlag, Berlin, Heidelberg, 1995. 
[21] H.B. Lawson and M.-L. Michelsohn, Spin geometry, Princeton University Press, Princeton, 1989.

[22] J.M. Lee and T.H. Parker, The Yamabe problem, Bull. Amer. Math. Soc., New Ser. 17(1) (1987), 37-91.

[23] S. Maier, Generic metrics and connections on Spin- and Spin ${ }^{c}$ manifolds, Comm. Math. Phys. 188(2) (1997), 407-437.

[24] K. Uhlenbeck, Generic properties of eigenfunctions, Amer. J. Math. 98(4) (1976), 1059-1078.

INSTITUT FUER MATHEMATIK

Universitaet Potsdam, Am Neuen Palais 10

14469 PoTSDAM

GERMANY

E-mail address: andreas.hermann@lmpt.univ-tours.fr

ReCEIVEd June 26, 2012 